\title{
A Practical Method for the Vinylation of Aromatic Halides using Inexpensive Organosilicon Reagents
}

\author{
Scott E. Denmark ${ }^{*}$ and Christopher R. Butler \\ Roger Adams Laboratory, Department of Chemistry, University of Illinois, Urbana, IL 61801
}

\begin{abstract}
The preparation of styrenes by palladium-catalyzed cross-coupling of aromatic iodides and bromides with divinyltetramethyldisiloxane (DVDS) in the presence of inexpensive silanolate activators has been developed. To facilitate the discovery of optimal reaction conditions, Design of Experiment protocols were used. By the guided selection of reagents, stoichiometries, temperatures, and solvents the vinylation reaction was rapidly optimized with three stages consisting of ca. 175 experiments (of a possible 1440 combinations). A variety of aromatic iodides undergo cross-coupling at room temperature in the presence of potassium trimethylsilanoate using $\mathrm{Pd}(\mathrm{dba})_{2}$ in DMF in good yields. Triphenylphosphine oxide is needed to extend catalyst lifetime. Application of these conditions to aryl bromides was accomplished by the development of two complementary protocols. First, the direct implementation of the successful reaction conditions using aryl iodides at elevated temperature in THF provided the corresponding styrenes in good to excellent yields. Alternatively, the use of potassium triethylsilanolate and a bulky "Buchwald-type" ligand allows for the vinylation reactions to occur at or just above room temperature. A wide range of bromides underwent coupling in good yields for each of the protocols described.
\end{abstract}

\section{Introduction}

Arylethenes (styrenes) are among the most important building blocks in organic synthesis. Substituted styrenes find widespread application in the synthesis of fine chemicals ${ }^{1}$ and polymers. ${ }^{2}$ Additionally, substituted styrenes serve as excellent substrates for synthetic endeavors because of the easily tunable electronic and steric properties of the alkene moiety. Many modern enantioselective reactions have been developed using styrenes as the test substrates such as inter alia asymmetric hydroformylation, ${ }^{3}$ hydroamination, ${ }^{4}$ dihydroxylation ${ }^{5}$ epoxidation, ${ }^{6}$ and carboamination. ${ }^{7}$ Moreover, styrenes find increasing application for the development of olefin metathesis, both as substrates and as ligands for metal catalysts. $^{8}$

Although styrenes are classically prepared using the strongly basic conditions of dehydrohalogenation or dehydration, ${ }^{9}$ the advent of metal-catalyzed, cross-coupling reactions has provided a range of milder methods for their preparation. This strategy involves the combination of an organometallic donor (generally a vinylmetallic species) with an organic acceptor (generally an aryl halide or pseudohalide) (Scheme 1).

Address Correspondence to: Professor Scott E. Denmark, 245 Roger Adams Laboratory, Box 18, Department of Chemistry, University of Illinois, 600 S. Mathews Ave., Urbana, IL 61801, tel: (217) 333-0066, FAX: (217) 333-3984, e-mail: denmark@ scs.uiuc.edu

Supporting Information Available: Detailed experimental procedures, full characterization of all products. This material is available free of charge via the Internet at http://pubs.acs.org. 
The simplest vinyl donor, ethylene, is primarily used on industrial scale to prepare substituted styrenes from aryl halides via the Heck reaction. ${ }^{10}$ Whereas ethylene is the most atom efficient vinyl donor, it requires special handling which is not generally amenable to laboratory scale synthesis. Thus, a range of organometallic vinyl donors that successfully transfer a vinyl unit to aryl halides have been developed over the past twenty years. These donors include boron-, ${ }^{11}$ tin-, ${ }^{12}$ and magnesium-based ${ }^{13}$ reagents and have been demonstrated to react with a range of organic acceptors. Boron-based reagents, including vinylboronic acid, ${ }^{11 a}$ its related esters ${ }^{11 \mathrm{~b}, \mathrm{c}}$ and cyclic anhydrides, ${ }^{11 \mathrm{~d}}$ as well as potassium trifluoroborates, ${ }^{11 \mathrm{e}-\mathrm{g}}$ cross-couple with a range of aryl bromides at elevated temperatures. Despite the utility of these vinylboron species, there are difficulties associated with their preparation. For example, the parent vinylboronic acid must be either trapped immediately as one of the aforementioned derivatives or freshly prepared prior to each use. ${ }^{11 a}$ Although the vinylboronic acid derivatives are significantly more stable and are commercially available, their greater $\cos { }^{14}$ likely reflects the problems associated with their preparation..$^{15}$ Additionally, these reagents often require elevated temperatures and/or higher pressures for reaction. ${ }^{11}$ The vinylboron reagents are generally used with aryl bromides, whereas the vinyltributyltin reagents react with a broader scope of electrophiles, including aryl iodides, aryl- and vinyl bromides, aryl and vinyl chlorides, and triflates, to afford high yields of the corresponding vinylated products. ${ }^{16}$ Unfortunately, vinyltributyltin suffers from the heavy metal toxicity generally associated with organotin species, ${ }^{17}$ which, taken together with its cost, ${ }^{18}$ can significantly deter its use on a larger scale. The Kumada-type coupling of vinylmagnesium bromide and chloride is more limited in the scope of electrophilic partner because of the basicity and nucleophilicity of the vinylating agent. For compatible substrates, however, good yields of the corresponding styrene are observed. ${ }^{13}$ Alternatively, dibromoethane has been used as a vinyl bromide precursor for the preparation of styrenes from arylboronic acids, in a process that inverts the acceptor/donor relationship. ${ }^{19}$

Each of these vinylmetallic reagents has one or more limitations. An ideal vinylation method would involve an inexpensive and non-toxic donor capable of transferring a vinyl group to a wide range of acceptors under mild conditions and be compatible with many functional groups.

\section{Background}

Silicon-based donors, first reported by Kumada and Tamao, ${ }^{20}$ offer a number of advantages compared to other organometallic reagents in palladium-catalyzed cross-coupling reactions. In particular, organosilicon reagents are non-toxic ${ }^{21}$ and the silicon containing by-products are easily removed. Moreover, the organosilicon subunit can be installed by a wide range of reactions, such that the cross-coupling event can be combined with other powerful carboncarbon bond forming processes, such as: ring-closing metathesis, ${ }^{22}$ silylformylation, ${ }^{23}$ and silylcarbocyclization. ${ }^{24}$ The initial reports of silicon-based, palladium-catalyzed crosscoupling reactions involved the use of highly-reactive, in-situ generated pentacoordinate silicon reagents such as fluorosilicates or bis(catechol)silicates. ${ }^{25}$ Since those reports, a variety of alternative organosilicon donors have been developed, including trimethyl- and difluoromethyl-alkenylsilanes developed by Hiyama. These donors successfully react with aryl iodides, although they suffered from low reactivity and water sensitivity, respectively. ${ }^{26}$ Alkenyltrialkoxysilane $\mathrm{s}^{27}$ and dimethylsilanols ${ }^{28}$ are inherently more stable, and, with proper activation (typically a fluoride source stemming from the strong affinity of fluoride for silicon $^{29}$ ), do participate in cross-coupling processes. tetra-Butylammonium fluoride trihydrate (TBAF) is the most commonly employed fluoride source because of its solubility in organic solvents.

Recently, the use of Brønsted base activation (that avoids the requirement for fluoride activation) of alkenyldimethylsilanols in cross-coupling reactions has been developed in these 
and other laboratories. ${ }^{30}$ Initially, potassium trimethylsilanoate $\left(\mathrm{KOSiMe}_{3}\right)$ served as the Brønsted base activator that facilitated the palladium-catalyzed cross-coupling between alkenyldimethylsilanols and aryl iodides. ${ }^{31}$ In addition, alkali carbonates ${ }^{30 \mathrm{a}}$ and alkali hydroxides ${ }^{30 \mathrm{~b}}$ are also competent bases for the cross-coupling reactions. More recently, alkali metal alkenyl-, aryl- and heteroaryldimethylsilanolates, prepared from the corresponding dimethylsilanols with a metal hydride, have been used directly in cross-coupling reactions without additional activators. ${ }^{32}$ Alkenylsilanolates cross-couple effectively with aryl iodides, bromides and chlorides, to afford excellent yields under mild reaction conditions ${ }^{33}$

The transfer of a simple vinyl group using organosilicon-based, palladium-catalyzed, crosscoupling was first demonstrated by Hiyama and co-workers. ${ }^{26}$ The combination of vinyltrimethylsilane and 1-iodonaphthalene provided the corresponding styrene in excellent yield. In this case, the silicon moiety was activated for vinyl transfer using tris(dimethylamino) sulfonium difluorotrimethylsilicate (TASF) and HMPA as the solvent. Jeffrey and co-workers have shown that a combination of potassium fluoride and tetra- butylammonium chloride $(\mathrm{TBACl})$ in toluene also promotes the vinylation reaction under milder conditions in excellent yields. ${ }^{34}$ Alternatively, methylvinylsiletane, $(\mathbf{1}$, Chart 1$)$ developed in these laboratories, reacts with a variety of aryl iodides under TBAF activation. ${ }^{35}$ These reactions proceed at room temperature, provide good to excellent yields and tolerate a broad range of functional groups. Subsequent mechanistic studies demonstrated that the combination of an alkenylsiletane and TBAF. $3 \mathrm{H}_{2} \mathrm{O}$ affords the unsaturated propylmethylsilanol in situ. ${ }^{36}$ Because a dialkylsilanol is the active coupling intermediate, it follows that the use of dimethylvinylsilanol (2), would eliminate the requirement for the preparation of the siletane. Although dimethylvinylsilanol can be prepared in situ by the hydrolysis of chlorodimethylvinylsilane (3), it spontaneously dimerizes upon concentration, to form divinyltetramethyldisiloxane (DVDS, 4, Scheme 2).

In view of the impracticality of using $\mathbf{2}$ directly, alternative organosilicon-based vinyl donors that generate dimethylvinylsilanol equivalents in situ must be considered (Chart 1). These donors include the aforementioned vinylsiletane $\mathbf{1}$, as well as disiloxanes and polyvinylsiloxanes,${ }^{37}$ all of which require fluoride activation.

With the exception of reagent $\mathbf{1}$, all of these compounds are commercially available., The poly (vinylsiloxanes) are inexpensive, stable, and widely available (Chart 1, 5-8) and are competent vinyl donors in fluoride-activated, palladium-catalyzed cross-coupling reactions with aryl iodides. ${ }^{37}$ Of the available polyvinylsiloxanes, $1,3,5,7$-tetravinyl-1,3,5,7-

tetramethylcyclotetrasiloxane $\left(\mathrm{D}_{4} \mathrm{~V}, \mathbf{5}\right)$ was chosen because of its low cost and high coupling efficiency, as each of the four vinyl groups is available for transfer. ${ }^{38} \mathrm{D}_{4}{ }^{\mathrm{V}}$ affords high yields of substituted styrenes from aryl iodides, and tolerates a range of functional groups and steric environments on the aryl halide. Recently, the scope of electrophiles available to couple with $\mathrm{D}_{4}{ }^{\mathrm{V}}$ has been extended to aryl bromides by employing an electron-rich sterically hindered phosphine ligand, 2-(di-t-butylphosphino)biphenyl (BPTBP, 10), to provide substituted styrenes in good to excellent yield (Scheme 3). ${ }^{39}$

Despite these advances, fluoride activation is still required. To overcome this limitation, we began to investigate a non-fluoride-based vinylation reaction. Specifically, our aim was to develop a method for the vinylation of aryl halides that entailed the use of: (1) an inexpensive and readily available vinylsilicon donor, (2) an inexpensive and readily available activator and (3) mild conditions that would be amenable to large-scale reactions. Our strategy was to initially develop conditions suitable for the vinylation of a range of aryl iodides and thereafter apply these conditions to aryl bromides (and chlorides) with modifications as needed.

By analogy with the non-fluoride activation of organodimethylsilanols, the obvious starting point for the current study would have been the examination of dimethylvinylsilanol as a vinyl 
donor but because this reagent is excluded (vide supra), our studies focused on two alternative approaches, (1) the use of the commercially-available, potassium dimethylvinylsilanolate (11, Scheme 4) and (2) the development of a novel method for generation of $\mathbf{1 1}$ from simple precursors. To expedite this discovery process, Design of Experiment (DoE) protocols were enlisted. ${ }^{40}$ The successful incorporation of this tool would allow for the examination of several reaction variables simultaneously and arrive at preparative conditions considerably faster than the standard individual- or parallel-strategy optimization.

\section{Results}

\section{Substrate Selection}

The investigation began with two orienting experiments that were guided by our existing knowledge of fluoride-free cross-coupling reactions. 4-Iodoanisole (12a) was chosen for the model study because of it is electron-rich, which should make the model cross-coupling reaction more representative of a larger scope of aryl iodides in contrast to choosing a more reactive, electron-deficient substrate. First, 12a was treated with $\mathrm{D}_{4}{ }^{\mathrm{V}}$ using 2 equiv of potassium trimethylsilanolate $\left(\mathrm{KOSiMe}_{3}, \mathbf{1 3}\right)$ as the activator in place of TBAF (Scheme 4, eq. 1). These conditions, which employ $\pi$-allylpalladium chloride dimer (APC) as the catalyst in THF, provided the corresponding styrene 14a in only $11 \%$ yield after $24 \mathrm{~h}$ at room temperature. Second, the viability of the commercially available potassium dimethylvinylsilanolate (11) was examined (Scheme 4, eq. 2). Unfortunately, even with 2 equiv of the silanolate, only a $9 \%$ yield of 14a was observed after $24 \mathrm{~h}$. A brief examination of $\mathrm{KOSiMe}_{3}$ as the activator and $\mathrm{Cy}_{3} \mathrm{P}$ as the ligand improved the yield of the reaction, but in no case was complete conversion observed. Therefore, it became clear that the simple adaptation of an existing method was not a feasible strategy to accomplish the fluoride-free vinylation.

These initial results suggested that the generation of an alkali dimethylvinylsilanolate was needed but not from deprotonation of the corresponding silanol. One such alternative was the in situ generation by the reaction of $\mathrm{KOSiMe}_{3}$ and DVDS via silanolate exchange (Scheme 5). The tendency for organodimethylsilanols to form disiloxanes is often an obstacle in the development of their cross-coupling reactions. Disiloxanes are not competent donors under non-fluoride reaction conditions, and their formation unproductively consumes two equiv of the starting silanol. Whereas disiloxane formation is generally a "dead-end" under standard Brønsted basic activation, silanolate exchange conditions would employ disiloxane $\mathbf{4}$ as a starting point. Moreover, the reaction should be close to thermoneutral, wherein one silanolate $\left(\mathrm{KOSiMe}_{3}\right)$ would react with a disiloxane (DVDS) to generate another silanolate (potassium dimethylvinylsilanolate) in addition to new, mixed disiloxane. The results of this study are compiled in Table 1.

The combination of 2.0 equiv each of $\mathrm{KOSiMe}_{3}$ and DVDS at room temperature provided a marked improvement over the previous conditions. Changing the palladium source provided only a marginal effect (entries 1 vs. 2 ) and increasing the temperature had no effect on the reaction (entry 2 vs. 3). A comparison of two ligands provided interesting results. Whereas the bidentate 1,4-bis(diphenylphosphino)butane (dppb) inhibited the reaction (entry 5), the bulky, electron-rich BPTBP markedly improved the yield (entry 4). Differences in relative stoichiometry between $\mathrm{KOSiMe}_{3}$ and DVDS improved the reaction significantly, but additional increases afforded no further benefit (entries 2 vs. 6 and 7).

The moderate improvements that accrued from this preliminary study made it clear that many variables influence the outcome of the reaction. Although we were confident that, given enough time and effort, a systematic investigation of each variable would provide suitable reaction conditions, we nevertheless chose to streamline our investigation by employing Design of Experiment (DoE) protocols. ${ }^{40}$ The implementation of DoE protocols would allow us to rapidly 
screen many reaction variables and determine their impact. Additionally, DoE protocols allow insight into the interaction of the reaction variables with one another.

\section{Application of Design of Experiment Protocols}

The DoE phase of the study was initiated by employing a rough D-optimal design, involving a limited number of reactions (24) but larger range of reaction variables (Table 2). ${ }^{41}$

In this design, six reaction factors were examined, including the choice of vinyl donor, activator, palladium source, ligand, and solvent. Additionally, the ligand loading was varied between 0,5 , and $10 \mathrm{~mol} \%$. On the basis of previous successes, $\mathrm{D}_{4}{ }^{\mathrm{V}}$, DVDS, vinyldimethylethoxysilane (DMVSOEt, 9), and hexavinyldisiloxane (HVDS, 8 ) were selected as potential vinyl donors. ${ }^{42}$ Potassium trimethylsilanolate (13) and cesium hydroxide (previously shown to promote the cross-coupling of aryldimethylsilanols) ${ }^{30}$ along with potassium hydroxide were chosen as activators. In all cases, 2.0 equiv of activator and 1.2 equiv of vinyl donor were used. Two palladium(II) sources (APC and $\mathrm{PdCl}_{2}$ ) and one palladium (0) source (bisdibenzylideneacetone palladium $\left[\mathrm{Pd}(\mathrm{dba})_{2}\right]$ ) were chosen, in combination with a range of ligands including dppb, the mono- and bisoxides of dppb (dppbO and $\mathrm{dppbO}_{2}$ ), $\mathrm{AsPh}_{3}$, and BPTBP. In each case, $5 \mathrm{~mol} \%$ of palladium was used, and the ligand was varied as necessary to examine the palladium/ligand ratio. Dioxane, THF, toluene and DMF were chosen as solvents. The reactions were performed (in an argon atmosphere) in a parallel fashion, using an 18-vessel carousel reactor, ${ }^{43}$ and were monitored by GC analysis at $0.5,3$, and $12 \mathrm{~h}$. The carousel was used to minimize variation in the reaction execution.

Overall, an improvement in the yield of 14a was observed. The 24 reactions had an average yield of $15 \%$, four of which gave no product. Comparing vinyl donors, DVDS, DMVSOEt, and HVDS each afforded yields greater than $28 \%$, whereas $\mathrm{D}_{4} \mathrm{~V}$ provided only $14-17 \%$ yield in the best cases. $\mathrm{KOSiMe}_{3}$ and $\mathrm{CsOH}$ were both superior to $\mathrm{KOH}$ (average yields using each reagent: $20 \%, 16 \%$, and $8 \%$, respectively). The palladium sources were equally delineated, as $\mathrm{PdCl}_{2}$ was inferior to both APC and Pd(dba) (average yields: $\mathrm{PdCl}_{2}-10 \%, \mathrm{Pd}(\mathrm{dba})_{2}-18 \%$, APC - 18\%). The reaction proceeded in all solvents tested, although DMF was slightly superior (average yields: toluene - 11\%, THF - 12\%, DMF - 20\%, dioxane - 15\%). The highest yield resulted from the combination of cesium hydroxide and DMVSOEt (9) in THF, with Pd $(\mathrm{dba})_{2} / \mathrm{AsPh}_{3}$ as the catalyst/ligand system, which afforded a $42 \%$ yield of $\mathbf{1 4 a}$ (GC analysis with internal standard). To verify the results, run \#6 was repeated in a standard reaction apparatus using a small increase in vinyl donor (2.0 equiv) and activator (3.0 equiv). After 12 $\mathrm{h}$, a $51 \%$ yield (GC) was observed.

Although only a modest improvement in the overall yield was observed the results pointed to two critical features that further directed our efforts. Most notable was the consistent failure of certain reagents, $\left(\mathrm{KOH}\right.$ and $\left.\mathrm{PdCl}_{2}\right)$ which were thus eliminated from future designs. ${ }^{44}$ Second, it was gratifying to observe that the most successful reaction involved a combination of reagents strikingly different than those used in the initial studies. These two features convinced us to undertake a more comprehensive optimization using DoE protocols.

Despite having eliminated some choices, a complete matrix of the remaining variables would have provided 1440 discrete and reasonable combinations. However, by employing DoE protocols for a second time, a set of 100 reactions that would provide the impact and key interactions of each of the seven categorical factors was identified. ${ }^{45} \mathrm{~A}$ second, more refined D-optimal design again examined vinyl donor $\left(\mathrm{D}_{4}{ }^{\mathrm{V}}\right.$, DVDS, DMVSOEt, HVDS), activator $\left(\mathrm{KOSiMe}_{3}\right.$ and $\mathrm{CsOH}$ ), solvent (DMF, THF, and dioxane), ligands (dppb, dppbO, dppbO $\mathrm{AsPh}_{3}$, and $\left.\mathrm{PPh}_{3}\right)$, palladium source ( $\mathrm{Pd}(\mathrm{dba})_{2}$ and $\left.\mathrm{APC}\right)$, ligand loading $(0,5$, and $10 \mathrm{~mol} \%)$. In this iteration, the role of the vinyl donor stoichiometry was also examined (1.2 and 2.4 vinyl equiv per iodide), whereas the first design had kept the vinyl donor stoichiometry constant. 
The overall goal of this design was simply the selection of optimal reagents. We hoped the randomization protocols used in the design would allow us to more quickly evaluate the candidates in each aspect of the transformation and the interactions of the various factors.

With the help of the reactor carousel and GC autosampler, the entire design was executed and analyzed in just over two weeks. Nine sets of conditions were identified that provided greater than $80 \%$ yield (GC analysis) at $12 \mathrm{~h}$ reaction times, (Table 3 ) including a best yield of $98 \%$ (Run 40). Moreover, the ligandless reaction of $\mathrm{KOSiMe}_{3}$ and DVDS in DMF with $\mathrm{Pd}(\mathrm{dba})_{2}$ as the catalyst was remarkably fast, providing an $88 \%$ yield of $\mathbf{1 4 a}$ within the first hour (run 7). More satisfying than the high yields, however, was the hierarchical delineation the data provided. From all of the results it was clear that $\mathrm{KOSiMe}_{3}$ was superior to $\mathrm{CsOH}$ (average yield: $\mathrm{KOSiMe}_{3}--42 \%$ vs. $\mathrm{CsOH}--20 \%$ ). Additionally, the reactions in DMF were superior to those in ethereal solvents in all head-to-head comparisons (average yields: DMF- 49\%, THF - 36\%, dioxane - 20\%). Equally striking was the failure of a majority of ligands for palladium, which appeared to slow the reaction considerably. Instead, reactions containing either a weakly stabilizing ligand $\left(\mathrm{dppbO}_{2}\right)^{46}$ or no ligand at all provided superior yields. Although $\mathrm{D}_{4} \mathrm{~V}$ consistently underperformed (average yield: 17\%), there was little difference between the remaining three vinyl donors (DVDS (39\%), HVDS (42\%), and DMVSiOEt (42\%)). In reactions that allowed for direct comparison, the yield for each donor was found to be within experimental error. Similarly, the two palladium sources differed only marginally (average yield: APC - 36\%, Pd(dba) $-31 \%)$.

From these results, the optimal activator $\left(\mathrm{KOSiMe}_{3}\right)$, solvent (DMF), and ligand $\left(\mathrm{dppbO}_{2}\right)$ could be identified. In contrast, whereas DVDS, DMVSOEt and HVDS were clearly superior to $\mathrm{D}_{4} \mathrm{~V}$, they were essentially indistinguishable from each other. To discriminate among these vinyl donors, as well as verify the results of the design, the donors were re-screened in bench top reactions using the conditions $\left(\mathrm{KOSiMe}_{3} / \mathrm{dppbO}_{2} / \mathrm{DMF}\right.$ ) suggested by the $\mathrm{DoE}$ experiments.

As seen in Table 4, the results were similar to those obtained from the DoE. Again, $\mathrm{D}_{4} \mathrm{~V}$ was inferior to the other vinyl donors (but did show an intriguing induction period) (entry 4). DVDS afforded the highest overall yield (95\%) and a significantly faster reaction (entry 2), although HVDS and DMVSOEt also provided respectable yields (87\% and $89 \%$, respectively). With both DVDS and DMVSOEt, product decomposition was observed to varying extents. On the basis of these results and cost considerations,${ }^{47}$ DVDS was chosen as the optimal vinyl donor.

To minimize costs, the use of triphenylphosphine oxide was evaluated as a replacement for $\operatorname{dppb}(\mathrm{O})_{2}$. The reactions behaved identically and $\mathrm{Ph}_{3} \mathrm{P}=\mathrm{O}$ was deemed to be a suitable, if not preferable, substitute (Scheme 6).

With a full set of reaction components in hand, a routine reaction optimization still remained. To facilitate this process, a third iteration of DoE protocols was designed to examine the optimal stoichiometries of the vinyl donor, activator, and triphenylphosphine oxide. This iteration consisted of 40 reactions selected using a central composite design, 20 with both $\mathrm{Pd}(\mathrm{dba})_{2}$ and APC, and was again monitored by GC analysis, at one- and three-hour time points. ${ }^{48}$ The ten highest yielding experiments are presented in Table 5.

To our delight, high yields (ranging from $76 \%$ to $97 \%$ ) were observed in all cases with an overall average yield of $81 \%$ at $1 \mathrm{~h}$ and $88 \%$ at $3 \mathrm{~h}$. The reactions using APC showed an average yield of $78 \%$ at $1 \mathrm{~h}$ and $88 \%$ at $3 \mathrm{~h}$, whereas those with $\mathrm{Pd}(\mathrm{dba})_{2}$ showed $84 \%$ at $1 \mathrm{~h}$ and $88 \%$ at $3 \mathrm{~h}$. From these results, there is still little difference between these two sources, though Pd $(\mathrm{dba})_{2}$ promoted slightly faster reactions. The impact of the vinyl groups and $\mathrm{KOSiMe}_{3}$ stoichiometry was less pronounced. The comparisons of vinyl stoichiometry to yield, and $\mathrm{KOSiMe}_{3}$ /vinyl donor ratio to yield are presented in Figure 1. 
Whereas the maximum yield of $\mathbf{1 4 a}$ was observed at a ratio of $\mathrm{KOSiMe}_{3} /$ vinyl group between 2 and 2.6 equiv, there was no obvious dependence on the DVDS stoichiometry. On the basis of these results, the optimal conditions were selected as follows: 3.5 equiv $\mathrm{KOSiMe}_{3}, 0.75$ equiv of DVDS, $5 \mathrm{~mol} \%$ of $\mathrm{Pd}(\mathrm{dba})_{2}, 5 \mathrm{~mol} \%$ of $\mathrm{Ph}_{3} \mathrm{P}=\mathrm{O}$ in DMF at room temperature. Moreover, these conditions translated well to a preparative scale $(2 \mathrm{mmol})$, affording an $81 \%$ yield of $14 a$ in $1 \mathrm{~h}$.

\section{Preparative Vinylations of Aryl lodides}

The optimized reaction conditions were general for a range of aryl iodides (Table 6). The electron-rich 4-iodoanisole and 2-iodoanisole substrates were transformed smoothly to the corresponding styrenes (entries 1,7) in 80\% and 68\% yield, respectively. Electron-deficient substrates, including ethyl 4-iodobenzoate, additionally reacted smoothly to provide the corresponding styrenes in 76-81\% yields (entries $\mathbf{2}, \mathbf{5}, \mathbf{6}$ ). The vinylation of the ethyl estercontaining substrate (entry 2 ) is notable, as the productive coupling out competes ester cleavage known to occur with $\mathrm{KOSiMe}_{3}$. Additionally, a variety of functional groups are well tolerated, including esters, nitro groups, and cyano groups. There appears to be little effect on the reaction yield due to steric or electron effects as all of the substrates described are between $68 \%$ and $81 \%$ yield. Further, the preparation of 1-vinylnaphthalene and 2-vinylanisole demonstrates tolerance of moderate steric hindrance in the reaction, without any deleterious effect on yield (entries 2 and 7). Groups bearing an acidic proton, including amines and secondary amides did not react. The successful examples illustrate the preparation of a range of substituted styrenes under very mild reaction conditions. It is important to note that these reactions exhibited a significant exotherm in some cases (for example, the internal temperature of the reaction of 12a warmed from $25^{\circ} \mathrm{C}$ to $52^{\circ} \mathrm{C}$ ) in the first 10 minutes of the reaction. Decreasing the catalyst loading attenuated the exotherm somewhat, but even using $2.5 \mathrm{~mol} \%$ palladium, a similar rise in temperature was observed for entry 2 . This was not the case for all substrates, however.

\section{Investigation into the Silanolate Exchange}

The silanolate exchange reaction between DVDS and $\mathrm{KOSiMe}_{3}$ is crucial for the efficient generation of the active dimethylvinylsilanolate nucleophile. Therefore, further investigation into the exchange was required to aid in further development of the vinylation chemistry. In an earlier study of the exchange process, a reaction temperature of $100{ }^{\circ} \mathrm{C}$ in toluene was required before rapid and complete exchange provided one equivalent of hexamethyldisiloxane and two equivalents of potassium dimethylvinylsilanolate as observed by ${ }^{18} \mathrm{O}-\mathrm{NMR}$ analysis (Scheme 7). ${ }^{49}$

To compare the results from this study with those described herein the silanolate exchange was examined in toluene and DMF and was monitored by ${ }^{1} \mathrm{H}-\mathrm{NMR}$ spectroscopy. In toluene, no exchange was observed between $\mathrm{KOSiMe}_{3}$ and DVDS at room temperature (Table 7, entry 1), but partial exchange was observed upon heating to $50{ }^{\circ} \mathrm{C}$ (entry 2). However, in DMF, nearly complete exchange occurs within $15 \mathrm{~min}$ at room temperature (entry 4). Because the two silanolates possess similar steric environments, the preference for formation of potassium dimethylvinylsilanolate and hexamethyldisiloxane at equilibrium is most likely due to electronic differences. Potassium trimethylsilanolate is more basic than the corresponding dimethylvinylsilanolate because of the presence of three donating alkyl groups, whereas the vinyl group is less donating. That this exchange is under equilibrium control was established by carrying out independent reactions that employed varying amounts of each reagent. In an experiment with a large excess $\mathrm{KOSiMe}_{3}$ (entry 4), nearly all of the DVDS was converted to the corresponding silanolate. In contrast, using an excess of DVDS (entry 6) consumes most, but not all, of the limiting $\mathrm{KOSiMe}_{3}$ implying that the basicities of the two silanolates are not very different. ${ }^{50}$ Employing nearly equimolar ratios of DVDS and $\mathrm{KOSiMe}_{3}$ converts nearly all of the DVDS to potassium dimethylvinylsilanolate. These exchange studies reinforce the 
results of the third DoE study, which showed that a large excess of potassium trimethylsilanolate and smaller excess of DVDS ( 0.75 equiv) are required.

\section{Vinylation of Aryl Bromides}

Although the results of the vinylation of aryl iodides were satisfying, the primary goal of this investigation was to develop a fluoride-free vinylation for aryl bromides. The challenge of engaging aryl bromides in palladium-catalyzed cross-coupling reactions arises from the greater $\mathrm{C}$-Br bond strength relative to that of aryl iodides. ${ }^{51}$ This difference is most pronounced in the oxidative addition step of the catalytic cycle of the cross-coupling reactions. The two most common strategies used to overcome this limitation are to raise the reaction temperature or to employ electron-rich, bulky phosphine ligands for palladium that lower the barrier required for oxidative addition.

To this end, a two-pronged strategy was developed that would both capitalize upon the results of the current study and exploit the wide range of ligands currently available for modulating the palladium center. Initially, the conditions developed for iodides were applied in a test case for the vinylation of 2-bromo-6-methoxynaphthalene, 17i (Scheme 8). By executing the reaction at $50^{\circ} \mathrm{C}, \mathbf{1 7 i}$ was smoothly converted to the corresponding styrene within $3 \mathrm{~h}$, to afford a $72 \%$ yield of $\mathbf{1 4 i}$.

Unfortunately, these conditions were not applicable to other aryl bromides. Incomplete reactions and irreproducible results were obtained in DMF at a range of temperatures. A significant amount of palladium black was observed in these reactions, most likely arising from insufficient stabilization of the catalyst. Moreover, dimethylamine was qualitatively observed upon completion of these reactions, likely due to the decomposition of DMF caused by $\mathrm{KOSiMe}_{3}$ at elevated temperatures. Therefore, the solvent was switched to THF to examine the vinylation of 4-bromoanisole, 17a, as it was comparable to DMF in the reaction with aryl iodides. Complete consumption of the bromide was observed in $6 \mathrm{~h}$ (at reflux), along with a $70 \%$ yield (GC) of 4-vinylanisole. ${ }^{52}$ Varying the DVDS stoichiometry did not improve the yield, although a slight increase in the amount of DVDS provided a faster reaction. These conditions were applicable on a preparative scale and were applied to a brief survey of aryl bromides (Table 8 ).

A variety of aryl bromides were tested in the coupling reaction and the corresponding styrenes were obtained in modest to excellent yield. Substrates bearing electron-withdrawing groups in the para- (entries 2-3 and 6) and ortho-positions (entry 7) provided reasonable to good yields of the corresponding styrenes. The reaction is compatible with some carbonyl groups, e.g. ketone (entry 3), although amide was less well tolerated (entry 6), and esters did not survive the coupling conditions, likely due to cleavage by $\mathrm{KOSiMe}_{3}$. It is worth emphasizing the successful coupling of 4-bromobenzophenone, as it demonstrated the mild reaction conditions associated with this protocol, whereas this substrate failed in the reactions in DMF. Some heterocycles are also well tolerated in the reaction, as evidenced by the high-yielding vinylation of 3-bromoquinoline (entry 4). Moreover, the applicability of this reaction to larger scale was demonstrated by the isolation of 3-vinylquinoline (entry 4) in $91 \%$ yield on a $20 \mathrm{mmol}$ scale.

This protocol was successful for a range of moderately activated aryl bromides. Although 4bromoanisole and 6-methoxynaphthyl bromide reacted smoothly, other electron rich bromides were less suitable. In addition, sterically-hindered substrates bearing electron-donating groups generally were recovered unreacted. These failures presumably stem from the inability to engage the aryl bromides in the oxidative addition step under these conditions. Increasing the reaction temperature afforded up to a $30 \%$ yield of styrenes in selected cases, indicating that oxidative addition was occurring. Unfortunately, the reactions never reached completion, most 
likely due to catalyst death, even in the presence of stabilizing ligands such as triphenylphosphine.

To address these shortcomings, new conditions that allowed the vinylation to occur at lower temperatures were needed. Under the assumption that the barrier to oxidative addition needed to be lowered, ligands that facilitate this key step at lower temperatures were tested. The study began by examining the vinylation of 4-bromoanisole (17a) using a series of dialkyl-2biphenylphosphine ligands developed by Buchwald and co-workers (Scheme 9). ${ }^{53}$ No reaction was observed using S-Phos or $\mathrm{Ph}_{2} \mathrm{X}$-Phos, although using ligand 20, a significant amount of aryl bromide was consumed at room temperature. In this case, 4-methoxydimethylaniline was formed from $\mathrm{C}-\mathrm{N}$ coupling of 17a with dimethylamine liberated by the decomposition of DMF, promoted by $\mathrm{KOSiMe}_{3}$. In the presence of BPTBP (10) no reaction was observed at room temperature. However, at $50{ }^{\circ} \mathrm{C}$ the aryl bromide was completely consumed and $\mathbf{1 4 a}$ was observed, although C-N coupling was again observed and was now the major product. At this point, it became clear that the small, nucleophilic $\mathrm{KOSiMe}_{3}$ was responsible for the unwanted C-N coupling byproducts (indirectly via promoting the decomposition of DMF), thereby stimulating the search for a different activator. ${ }^{54}$

\section{Potassium Dimethylvinylsilanolate as Vinyl Donor}

The most straightforward solution to replace $\mathrm{KOSiMe}_{3}$ was to eliminate the requirement for activation by employing the pre-formed potassium dimethylvinylsilanolate (11). Although only traces of product were seen using commercially available 11, no C-N coupling was observed. To avoid the possibility that these results were caused by impurities in the commercial material, $\mathbf{1 1}$ was prepared by the one-pot conversion of chlorodimethylvinylsilane to the silanol and subsequent deprotonation to afford a $34 \%$ yield on a 5 -g scale (Scheme 10). 55

Using prepared 11, productive vinylation of $\mathbf{1 7 a}$ was observed at room temperature without C-N coupling (Table 9, entry 1). Increasing the number of equivalents of silanolate had little effect on the yield, whereas increasing the temperature improved the conversion (entries 2 and 3). Concomitant increases in temperature and stoichiometry provided a $63 \%$ yield of $\mathbf{1 4 a}$ in 2 $\mathrm{h}$, but no further conversion was observed (entry 4$)$. The remainder of the mass balance was unreacted 17a.

Incomplete reaction can result from a number of factors including disiloxane formation (observed) which would consume 11. Although the conversion could be increased by employing 4.0 equiv of the silanolate, complete conversion of the aryl bromide was never observed. To suppress the disiloxane formation, the sodium dimethylvinylsilanolate and the potassium diisopropylvinylsilanolate were prepared to alter the nucleophilicity and steric environment of the vinylsilanolate, respectively. With sodium dimethylvinylsilanolate, no reaction was observed under a variety of conditions, whereas the use of potassium diisopropylvinylsilanolate at $60^{\circ} \mathrm{C}$ in THF with 10 provided only small amounts of $\mathbf{1 4 a}$. Thus, use of a pre-formed vinylsilanolate was not the answer.

\section{Potassium Triethylsilanolate as an Activator}

At this point the successful vinylation of electron-rich or sterically hindered aryl bromides was stymied by two different problems. The successful coupling of these substrates with $\mathbf{1 1}$ in the presence of $\mathbf{1 0}$ demonstrated that a judicious choice of ligands was crucial, but also that disiloxane formation must be avoided. On the other hand, disiloxane formation was not a problem with $\mathrm{KOSiMe}_{3} / \mathrm{DVDS}$, but undesired C-N coupling predominated in the presence of ligand 10. Thus, the silanolate exchange process was revisited with the objective of identifying a new activator for DVDS that was capable of facilitating the silanolate exchange (thus 
generating 11 in situ) while avoiding the preferential C-N coupling associated with $\mathrm{KOSiMe}_{3}$.

A suitable activator should exhibit the following features: (1) it should contain no transferable groups, (2) it should exhibit similar electronic properties to $\mathrm{KOSiMe}_{3}$ and facilitate silanolate exchange, but (3) it should be bulkier than $\mathrm{KOSiMe}_{3}$ to prevent loading on the $\operatorname{ArylPdL}_{\mathrm{n}} \mathrm{X}$ intermediate, and (4) it should not decompose DMF at temperatures required for vinylation. With these criteria in mind, potassium triethylsilanolate $\left(\mathrm{KOSiEt}_{3}, \mathbf{2 1}\right)$ was selected for testing. The preparation of $\mathrm{KOSiEt}_{3}$ began with the buffered hydrolysis of commercially available chlorotriethylsilane (22), to afford triethylsilanol (23) (Scheme 11). Unlike trimethyl- and dimethylvinylsilanol which were not isolable because of their rapid dimerization, triethylsilanol was obtained in $95 \%$ yield upon distillation. The silanol was then treated with potassium hydride in THF to afford an $84 \%$ yield of $\mathrm{KOSiEt}_{3}(\mathbf{2 1})$ as a white powder.

As alluded to above, for $\mathrm{KOSiEt}_{3}$ to be an effective activator it must exchange with DVDS. In THF, the exchange was complete within 15 minutes by ${ }^{1} \mathrm{H}-\mathrm{NMR}$ analysis. ${ }^{56}$ Interestingly, only one exchange was observed, in which the mixed disiloxane and 1.0 equiv of the dimethylvinylsilanolate were formed (Scheme 12, equation 1). However, in DMF, the exchange was rapid and complete as was observed for $\mathrm{KOSiMe}_{3}$ (equation 2).

The first cross-coupling experiment employed $\mathrm{KOSiEt}_{3}$ (2.8 equiv), DVDS (1.2 equiv) and 17a with $2.5 \mathrm{~mol} \%$ of APC and $5 \mathrm{~mol} \%$ of 10 at $60{ }^{\circ} \mathrm{C}$ in DMF. ${ }^{57}$ This reaction provided a $63 \%$ yield (GC) of the corresponding styrene within $30 \mathrm{~min}$ with no bromide remaining (Scheme 13). More gratifying was the observation that the reaction proceeded at room temperature (54\% in $24 \mathrm{~h}$ ). Other ligands did not improve the reaction; the use of X-Phos provided a 53\% yield in $2 \mathrm{~h}$ and S-Phos was completely ineffective. Interestingly, under no conditions was the product of $\mathrm{C}-\mathrm{N}$ coupling detected, further supporting the hypothesis that $\mathrm{KOSiMe}_{3}$ was responsible for these side products.

To improve the amount of productive coupling, different stoichiometries of $\mathrm{KOSiEt}_{3}$ and DVDS were examined at $50^{\circ} \mathrm{C}$ (Table 10). Increasing the amounts of DVDS or KOSiEt 3 alone had no overall effect (entries 1 vs. 4, 2 vs. 5), whereas increasing the ratio of $\mathrm{KOSiEt}_{3}$ to DVDS markedly increased the initial reaction rate but not the overall yield (entries 1 vs. 2). In contrast, the reaction temperature did influence the yield of the coupling. Decreasing the temperature from $50{ }^{\circ} \mathrm{C}$ to $23{ }^{\circ} \mathrm{C}$ afforded a $77 \%$ yield of the corresponding styrene after $12 \mathrm{~h}$, although $15 \%$ of the starting material remained. A slight increase to $35^{\circ} \mathrm{C}$ with a concomitant increase in $\mathrm{KOSiEt}_{3}$ (4.0 equiv) provided an $85 \%$ yield (GC) with complete consumption of starting material after $4 \mathrm{~h}$. Under these conditions, 14a was isolated in $74 \%$ yield on a $2.0 \mathrm{mmol}$ scale.

\section{Preparative Vinylation of Aryl Bromides}

A series of aryl bromides was surveyed to test the generality of these vinylation conditions, (Table 11). The substrates examined represented a range of electronic and steric influences and contained a number of sensitive functional groups. Aryl bromides with substituents in the 4position worked well, affording the corresponding styrenes in 50-74\% yield (entries 1-5). Electron-rich aryl bromides including those bearing methoxy- or dimethylamino- substituents worked well (72-74\% yields, entries 1, 2, and 7). Similarly, electronically neutral substrates such as 1-bromonaphthalene or 3-bromoquinoline also provided good yields of the corresponding styrenes (entries 9 and 11). Electron-deficient substrates provided diminished yields likely due to competitive anionic polymerization of the corresponding styrene (entries 3-5). Steric hindrance does not interfere with the reaction, as evidenced by the preparation of 2-vinylmesitylene and 2-vinylanisole (entries 7, 8). A variety of functional groups are well tolerated, including amides, amines, ethers, esters, and chlorides. Although these reaction 
conditions caused the cleavage of a TBS ether, replacing DMF with THF allows the styrene to be formed in good yield without deprotection (entry 6).

Despite the successful vinylation of a variety of bromides, the reaction is not without limitations. The substrates in Chart 2 did not afford the corresponding styrenes using the developed reaction conditions. First, substrates bearing acidic hydrogens are not tolerated in the reaction. Second, styrenes bearing strongly electron-withdrawing groups, such as ketones, polymerized under the reaction conditions, whereas they were tolerated in the reactions in THF. Moreover, hindered substrates containing a Lewis basic site failed in the reaction including the $\mathrm{N}$-benzylpyrazole and 2-(dimethylamino)bromobenzene.

\section{Discussion}

The primary goal of this project, namely the development of a non-fluoride-based vinylation of aryl halides, required a method for the generation of a dimethylvinylsilanolate salt in situ. Unlike the coupling of alkenyldimethylsilanols, which are stable, storable substances, the parent dimethylvinylsilanol is too prone to dimerization to make it a useful reagent.

\section{Design of Experiment Protocols}

Because the parameters for a successful reaction were unknown, the DoE process was initiated with a wide array of variables, including those that had not worked in any previous cases. The first iteration of the DoE plan was designed to refine the selection of variables and eliminate those that showed no promising results. The concept was successful, as $\mathrm{KOH}, \mathrm{PdCl}_{2}$ and toluene were each eliminated given their low success rate, thus allowing fewer variables to be studied with greater frequency in the second design.

The second iteration of DoE was designed with the intent of reagent selection. Whereas DoE protocols are used routinely in the optimization of reaction conditions, there are fewer examples that employ DoE protocols for reactions development, i.e. reagent selection. In this regard, the success of the design was mixed, as the superior activator $\left(\mathrm{KOSiMe}_{3}\right)$, solvent (DMF), ligand $\left(\mathrm{dppbO}_{2}\right)$ and vinyl donor stoichiometry (2.0 equiv) were clearly identified. However, the identification of optimal vinyl donor, palladium source, and ratio of ligand to palladium was not achieved. Although it was satisfying that three of the four vinyl donors afforded high yields, further investigation was required to distinguish the three. Similarly, there was no distinction provided between APC and $\mathrm{Pd}(\mathrm{dba})_{2}$. A further shortcoming of the design involved establishing the ligand/palladium ratio. In part, this failure could be attributed to the nature of the optimal bisphosphine bisoxide ligands, known in related systems to prevent catalyst decomposition pathways. Error! Bookmark not defined. Despite these inconclusive aspects, the overall benefit of the design was seen in the increase in yield from $43 \%$ to $95 \%$.

The third iteration of the DoE involved the more common optimization of reagent stoichiometry. A central composite design was selected because of its ability to rapidly accomplish this optimization with good statistical relevance. Owing to the high yields (>90\%) already observed, center points could be selected with a reasonable amount of confidence that optimal conditions would be found. This choice assumes that the optimal stoichiometry is not much different from the conditions at hand. This assumption proved valid, as the optimal conditions were easily identified. More importantly, DoE protocols allow for higher-order mathematical analyses. From the quadratic model generated by the DoE software, the yields at each time point can be visualized in three dimensions. These graphs (Figure 2) demonstrate the interaction of the following factors: (1) the ratio of $\mathrm{KOSiMe}_{3}$ to vinyl group (x-axis), (2) equivalents vinyl group (y-axis), and (3) yield of 14a (\%, z-axis). Each surface model represents a given time point $(1 \mathrm{~h}$ and $3 \mathrm{~h})$ and is delineated by the palladium source. 
Two aspects of the reaction are immediately apparent from the above graphs. The first is the difference in reaction rates between $\mathrm{APC}$ and $\mathrm{Pd}(\mathrm{dba})_{2}$. The reactions that use $\mathrm{Pd}(\mathrm{dba})_{2}$ are apparently faster than those using APC, but only at higher $\mathrm{KOSiMe}_{3} /$ vinyl ratios. Despite the high initial rates, the APC reaction proceeds to similar yields within three hours. Most likely, this difference in rate is related to the reduction of palladium(II) to palladium(0). ${ }^{58}$ The second is the difference in the factors that significantly impact the reaction for the different palladium sources. In the reactions that employ APC, the number of vinyl equivalents moderately impacts the yield (Figure 2c), whereas the changing the ratio of $\mathrm{KOSiMe}_{3}$ to vinyl groups has little effect. In contrast, the yields of the reactions employing $\mathrm{Pd}(\mathrm{dba})_{2}$ are more greatly influenced by the ratio of $\mathrm{KOSiMe}_{3}$ to vinyl groups (Figure 2b, 75\% yield with 1.41 equiv $\mathrm{KOSiMe}_{3} /$ vinyl group vs. $>90 \%$ yield with 2.59 equiv $\mathrm{KOSiMe}_{3} /$ vinyl group), whereas increasing the number of vinyl equivalents has a minimal effect at the $1 \mathrm{~h}$ time point. Moreover, at $3 \mathrm{~h}$, there appears to be deleterious effect of employing high levels of both $\mathrm{KOSiMe}_{3}$ and DVDS, as the yield diminished in the top corner in comparison to the $1 \mathrm{~h}$ yield.

In contrast to the subtle differences between the palladium sources, the second DoE provided clear distinction among the solvents employed, e. g. DMF promoted the reaction to a much greater extent than ethereal solvents. This difference is likely related to a solvent effect on the rate of silanolate exchange between DVDS and $\mathrm{KOSiMe}_{3} .{ }^{59}$ Silanolate exchange can take place by either direct displacement or by formation of a hypercoordinate siliconate complex. By either mechanism, a change to a more polar solvent is expected to accelerate the rate of displacement and thereby the rate at which the equilibrium is established.

\section{Preparative Vinylation of Aryl lodides}

Application of the optimized conditions ( $\mathrm{KOSiMe}_{3}$ (3.5 equiv), DVDS ( 0.75 equiv), Pd $(\mathrm{dba})_{2}(5 \mathrm{~mol} \%), \mathrm{Ph}_{3} \mathrm{P}=\mathrm{O}(5 \mathrm{~mol} \%)$, DMF, rt) to a range of aryl iodides provided good to excellent yields of the corresponding styrenes. Although the reaction had been optimized for 4-iodoanisole, electron deficient aryl iodides also worked well. It is notable that the vinylation of ethyl 4-iodobenzoate is not complicated by ester cleavage with $\mathrm{KOSiMe}_{3}{ }^{60}$ Unfortunately, the reaction of the corresponding methyl 2-iodobenzoate does suffer ester cleavage, as 2iodobenzoic acid is produced. Also notable is the high yield of the electron-deficient 4-cyanoand 3-nitrostyrenes despite their propensity for anionic polymerization. ${ }^{61}$ Although the yields may be somewhat lower than those obtained by other methods, they are still above $68 \%$ without optimization. Moreover, the goal of identifying inexpensive reagents for the vinylation was accomplished, as the palladium catalyst is now the most expensive component in the reaction. Additionally, the reagents require no special handling or preparation, and are non-toxic, which makes them suitable for large-scale applications.

\section{Vinylation of Aryl Bromides}

Adapting the conditions developed for aryl iodides to the vinylation of aryl bromides was initially envisioned to involve two different strategies. The slower oxidative addition to bromides compared to iodides was to be overcome by increasing the reaction temperature or by the use of bulky, electron-rich phosphine ligands. Higher temperatures did induce the vinylation of both electron-deficient and sterically unencumbered aryl bromides.

Unfortunately, these conditions failed to engage electron-rich or sterically congested aryl bromides. The use of bulky phosphine ligands such as $\mathbf{1 0}$ provided either no reaction (at room temperature) or the products of C-N coupling at elevated temperatures.

These failures were difficult to reconcile. The vinylation of aryl iodides using $\mathrm{KOSiMe}_{3} / \mathrm{DVDS}$ demonstrated that the silanolate exchange occurred rapidly at room temperature and therefore could not be responsible for the failure of the reactions with aryl bromides. Moreover, the alkenylation of aryl chlorides in the presence of ligands 18 or $\mathbf{1 9}^{33}$ suggested that once $\mathbf{1 1}$ was 
bound to the palladium center, a pathway should be available for transmetalation. The vinylation of 4-bromoanisole with preformed $\mathbf{1 1}$ (in the presence of $\mathbf{1 0}$ at room temperature) confirmed the viability of the vinylation pathway. However, no conditions could be found in which the bromide was completely consumed, because of competitive formation of DVDS from 11. Despite the lack of a proton source, disiloxane formation can occur by disproportionation of $\mathbf{1 1}$ with the polysiloxane generated throughout the reaction, providing an alternative pathway for silanolate consumption (Scheme 14, $\mathbf{E} \rightarrow \mathbf{4}$ ).

Apparently, $\mathrm{KOSiMe}_{3}$ is competitively binding to the palladium center and that the exchange of silanolates at palladium must not be possible at room temperature in the presence of $\mathbf{1 0}$. If this exchange were feasible, intermediates bearing 11 would form the product. The successful vinylation promoted by $\mathrm{KOSiEt}_{3}$ with DVDS supported this hypothesis. A bulkier activator was expected to enable silanolate exchange, but not compete with $\mathbf{1 1}$ for binding to the arylpalladium halide, thus resulting in the desired cross-coupling.

Optimization of the reactions with $\mathrm{KOSiEt}_{3}$ as the activator revealed a few interesting features. The significant rate change observed when $\mathrm{KOSiEt}_{3}$ or DVDS are independently varied is likely related to the silanolate exchange process, where reversible inhibition of the coupling (initially observed with the aryl iodides) arises from the Pd(0)-ligating ability of DVDS. Thus, when insufficient amounts of $\mathrm{KOSiEt}_{3}$ are present to convert all of the DVDS to the silanolate, the remaining DVDS interferes by sequestering the palladium(0).

The application of the optimal reaction conditions proved to be general for a range of electronrich, electron-neutral, and moderately electron-deficient aryl bromides. Broad functional compatibility was observed due to the relatively mild reaction conditions. Aryl bromides bearing strongly electron-withdrawing substituents including carbonyl and nitro groups fail in the reaction, likely due to anionic polymerization of the styrene facilitated by $\mathrm{KOSiEt}_{3}$ at elevated temperatures.

\section{Unified Mechanistic Picture}

From the analysis above, a mechanism for the entire process can now be formulated (Scheme 15). This scheme, shown previously as Scheme 12, can now be analyzed in detail for the consequences of activator structure, aryl halide, temperature, and ligands. Each step will be discussed below in the context of the entire body of results.

(a) Silanolate Exchange-Silanolate $\mathbf{1 1}$ can be generated in situ by equilibrium redistribution of DVDS with a trialkylsilanolate. Both $\mathrm{KOSiMe}_{3}$ and $\mathrm{KOSiEt}_{3}$ can establish this equilibrium, albeit at different rates and with different stoichiometries, however both are rapid under the conditions of the coupling.

(b) Oxidative Addition-Insertion of palladium(0) into a carbon-iodine bond occurred at room temperature with the aid of a phosphine oxide to stabilize the palladium catalyst. However, for aryl bromides, bulky phosphine ligands were needed to facilitate oxidative addition to provide palladium(II) intermediate $\mathbf{A}$.

(c) Partitioning of Intermediate $\mathbf{A}$-Intermediate $\mathbf{A}$ is formed in a rapid, irreversible step and can then react with either silanolates $\mathrm{KOSiR}_{3}$ or $\mathbf{1 1}$ generated by the equilibrium established in step a. Combination of $\mathbf{A}$ with $\mathbf{1 1}$ would produce the complex $\mathbf{B}$, whereas combination with either activator $\mathrm{KOSiMe}_{3}$ and $\mathrm{KOSiEt}_{3}$ would produce intermediate $\mathbf{C}$, which is believed to be unproductive under the reaction conditions. Alternatively, intermediate A can also combine with dimethylamine in reactions using $\mathrm{KOSiMe}_{3}$ in DMF at elevated temperature. ${ }^{62}$ From the results of room temperature couplings we conclude that with $\mathrm{KOSiEt}_{3}$, intermediate $\mathbf{B}$ predominates, whereas with $\mathrm{KOSiMe}_{3}$, intermediate $\mathbf{C}_{\mathbf{M}}$ 
predominates. Although these assertions seems reasonable on the basis of the differences in steric bulk, to better understand the basis for these conclusions, we must look for evidence further in the catalytic cycle below.

(d) Exchange between B and C-This critical equilibrium is highly dependent on the nature of the activator, the temperature and the presence or absence of bulky phosphine ligands. For $\mathrm{KOSiMe}_{3}$ the equilibrium is believed to be rapid at room temperature in the absence of phosphine ligands as evidenced by the successful vinylation of aryl iodides. However, this exchange does not occur readily at room temperature when bulky ligands are present because no reaction is observed with aryl bromides in the presence of $\mathbf{1 0}$ (thereby substantiating the overwhelming population of $\mathbf{C}_{\mathbf{M}}$ ) ${ }^{63}$ Exchange likely takes place at elevated temperatures as cross-coupling (from $\mathbf{B}$ ) is observed. For $\mathrm{KOSiEt}_{3}$, this equilibrium is not believed to occur because at all temperatures studied productive vinylation was observed. Because vinylation occurs at room temperature, it is believed that either: (1) $\mathrm{KOSiEt}_{3}$ cannot effectively compete with 11 for binding to the palladium center, or (2) if $\mathrm{KOSiEt}_{3}$ does bind, exchange with $\mathbf{1 1}$ is available.

(e) Cross-coupling-This process can take place at room temperature from aryl iodides and aryl bromides with preformed $\mathbf{1 1}$ or with a suitable method for its in-situ generation.

(f) C-N coupling - The C-N coupling process is observed in DMF with $\mathrm{KOSiMe}_{3}$ at elevated temperatures from the decomposition of DMF. Because the productive vinylation can take place at lower temperatures $\left(35-40{ }^{\circ} \mathrm{C}\right)$ with $\mathrm{KOSiEt}_{3}$, competitive $\mathrm{C}-\mathrm{N}$ coupling is not observed with this activator.

The formulation of this unified mechanistic scheme allows for a clear illustration of the challenges associated with developing a clean, general and reproducible vinylation with organosilicon reagents. The disparate behavior of the different activators, halides, and ligand sets can now be more clearly understood and nearly all of the data interpreted in the context of this framework. Thus, the superiority of $\mathrm{KOSiEt}_{3}$ compared to $\mathrm{KOSiMe}_{3}$ as an activator for the vinylation can be ascribed to a number of different factors. First, it is capable of generating 11 at room temperature from DVDS in reasonable concentration. Second, because of its bulk, it does not effectively compete with $\mathbf{1 1}$ for occupancy on the arylpalladium center, thus allowing the productive coupling to occur at lower temperature. Finally, because coupling can take place a lower temperature, the decomposition of DMF does not interfere.

\section{Conclusion}

Mild, fluoride-free reaction conditions for the vinylation of aryl iodides have been developed via a silanolate exchange reaction. The utilization of Design of Experiment protocols allowed for the examination of multiple variables simultaneously and facilitated rapid formulation reaction conditions for aryl iodides. The adaptation of these conditions to aryl bromides, based on mechanistic experiments, has provided a new, mild alternative for the preparation of substituted styrenes.

\section{Experimental Section}

\section{Representative General Experimental (See Supporting Information)}

Preparation of 4-Ethenylanisole from 4-lodoanisole using $\mathrm{KOSiMe}_{3}(14 \mathrm{a})$-In a dry box, $\mathrm{KOSiMe}_{3}$ (Aldrich, ${ }^{52} 896 \mathrm{mg}, 7.0 \mathrm{mmol}, 3.5$ equiv) was added to an oven-dried, twonecked, $10-\mathrm{mL}$, round-bottomed flask. The flask was removed from the dry box and Pd (dba) 2 ( $57 \mathrm{mg}, 0.1 \mathrm{mmol}, 0.5$ equiv), triphenylphosphine oxide ( $29 \mathrm{mg}, 0.1 \mathrm{mmol}, 0.05$ equiv) and 4-iodoanisole ( $468 \mathrm{mg}, 2 \mathrm{mmol}$ ) were added. The flask was equipped with an argon inlet 
adapter capped with a septum and evacuated. The flask was then refilled with argon, and this evacuation/refill process was repeated two additional times. The septum was then pierced and a thermocouple was inserted into the flask. DMF $(2.0 \mathrm{~mL})$ and DVDS $(344 \mu \mathrm{L}, 1.5 \mathrm{mmol}, 0.75$ equiv) were then added sequentially via syringe, followed by the remaining DMF ( $2.0 \mathrm{~mL})$. The reaction mixture was then stirred at $\mathrm{rt}$ and the internal temperature was observed to rise from $25{ }^{\circ} \mathrm{C}$ to $52{ }^{\circ} \mathrm{C}$ over $10 \mathrm{~min}$. The mixture was allowed to stir for $1.5 \mathrm{~h}$, and then was partitioned between pentane $(50 \mathrm{~mL})$ and water $(100 \mathrm{~mL})$. The aqueous layer was extracted with pentane $(2 \times 50 \mathrm{~mL})$, and the organic layers were sequentially washed with water (30 $\mathrm{mL}$ ). The organic layers were combined and dried over $\mathrm{Na}_{2} \mathrm{SO}_{4}$, filtered, and concentrated in vacuo. Purification by column chromatography (silica gel; $30 \mathrm{~mm} \times 110 \mathrm{~mm}$; pentane/ $/ \mathrm{Et}_{2} \mathrm{O}$, 100/1) afforded $215 \mathrm{mg}(80 \%)$ of $\mathbf{1 4 a}$ as a clear, colorless oil. Data for 14a: ${ }^{\mathbf{6 4}} \mathrm{H}$ NMR: (500 $\left.\mathrm{MHz}, \mathrm{CDCl}_{3}\right)$ 7.37(dd, $J=6.9$ and 1.9, $2 \mathrm{H}$ ), $6.88(\mathrm{dd}, J=6.8$ and 1.9, $2 \mathrm{H}), 6.68(\mathrm{dd}, J=17.6$ and 10.7, $1 \mathrm{H}), 5.63(\mathrm{~d}, J=17.8,1 \mathrm{H}), 5.14(\mathrm{~d}, J=11.0,1 \mathrm{H}), 3.81(\mathrm{~s}, 3 \mathrm{H}) ;{ }^{13} \mathrm{C}$ NMR: $(125$ $\mathrm{MHz},\left(\mathrm{CDCl}_{3}\right)$ 159.3, 136.2, 130.4, 127.3, 113.9, 111.5, 55.2; IR: 3087, 3004, 2956, 2935 , 2908, 2836, 1628, 1608, 1575, 1511, 1461, 1443, 1408, 1320, 1302, 1250, 1175, 1114, 1040, 1024, 991, 901, 835, 815, 709; MS: (EI, 70eV) 134, 119, 91, 65; $R_{f} 0.36$ (pentane/ Et $2 \mathrm{O}, 50: 1$ ).

\section{Preparation of 4-Ethenylbenzophenone from 4-Bromobenzophenone using}

$\mathrm{KOSiMe}_{3 .}$ (14k)-To an oven-dried, 1-necked, 10-mL, round-bottomed flask equipped with magnetic stir bar and a side-arm gas adapter fitted with a septum was added $\mathrm{KOSiMe}_{3}$ (Aldrich, $52896 \mathrm{mg}, 7 \mathrm{mmol}, 3.5$ equiv), $\mathrm{Pd}_{2}(\mathrm{dba})_{3}(58 \mathrm{mg}, 0.1 \mathrm{mmol}, 0.05$ equiv) and

triphenylphosphine oxide ( $28 \mathrm{mg}, 0.1 \mathrm{mmol}, 0.05$ equiv). The gas adapter was attached to an argon manifold, and the flask was evacuated and refilled with argon. This evacuation/refill process was repeated two additional times and then the flask was charged with THF ( $4 \mathrm{~mL})$ via syringe. The gas adapter was quickly removed, 4-bromobenzophenone (522 $\mathrm{mg}, 2 \mathrm{mmol}$ ) was added as a solid and the adapter replaced. DVDS ( $500 \mu \mathrm{L}, 2.2 \mathrm{mmol}, 1.1$ equiv) was then added neat, via syringe and the mixture was placed in a preheated oil bath and was heated to reflux for $3 \mathrm{~h}$. After the reaction was determined to be complete by GC analysis, the flask was cooled to rt, then was filtered through a small pad of silica gel $(5 \mathrm{~g})$. The silica plug was rinsed with $50 \mathrm{~mL}$ of $\mathrm{Et}_{2} \mathrm{O}$, and the filtrate was concentrated in vacuo. Purification of the crude product by column chromatography (silica gel; $30 \mathrm{~mm} \times 120 \mathrm{~mm}$ pentane/Et ${ }_{2} \mathrm{O} ; 20: 1$ ) afforded 386 $\mathrm{mg}(93 \%)$ of $\mathbf{1 4 k}$ as a white solid. Data for $\mathbf{1 4 k}:{ }^{27 \mathrm{~b}}{ }^{1} \mathrm{H}$ NMR: $\left(500 \mathrm{MHz}, \mathrm{CDCl}_{3}\right)$ 7.81-7.78 (m, $4 \mathrm{H}), 7.59$ (t, $J=7.6,1 \mathrm{H}), 7.49(\mathrm{~d}, J=8.3,2 \mathrm{H}), 7.48$ (t, $2 \mathrm{H}, J=7.6), 6.78$ (dd, $J=17.6$ and $10.8,1 \mathrm{H}), 5.90(\mathrm{~d}, J=17.6,1 \mathrm{H}), 5.41(\mathrm{~d}, J=10.8,1 \mathrm{H}) ;{ }^{13} \mathrm{C}$ NMR: $(125 \mathrm{MHz}$, $\left(\mathrm{CDCl}_{3}\right)$ 195.9, 141.3, 137.5, 136.4, 135.7, 132.0, 130.3, 129.7, 128.0, 125.8, 116.3; IR: 3082, 3060, 1927, 1830, 1657, 1577, 1604, 1555, 1447, 1403, 1317, 1278, 1177, 1148, 1116, 1073, 1027, 1000, 990, 938, 924, 857, 797, 789, 755, 703, 681; MS: (EI, 70eV) 209, 208, 132, 131, $105,103,77 ; R_{f} 0.37$ (hexane/EtOAc, 20:1).

\section{Preparation of 2-Ethenyl-6-methoxynaphthalene from 2-Bromo-6- \\ methoxynaphthalene using $\mathrm{KOSiEt}_{3}$. (14i)-To an oven-dried, 2-necked, 10-mL,} round-bottomed flask equipped with magnetic stir bar, septum and gas adapter was added allylpalladium chloride dimer ( $18 \mathrm{mg}, 0.05 \mathrm{mmol}, 0.025$ equiv), and 2-(di-t-butylphosphino) biphenyl ( $29 \mathrm{mg}, 0.1 \mathrm{mmol}, 0.05$ equiv). The gas adapter was attached to an argon manifold, and the flask was evacuated and refilled with argon. This evacuation/refill process was repeated two additional times, and the flask was then charged with DMF $(2 \mathrm{~mL})$ via syringe. The argon adapter was removed, and 2-bromo-6-methoxynaphthalene (474 $\mathrm{mg}, 2 \mathrm{mmol}$ ) was quickly added as a solid, and the gas adapter was replaced. To the mixture was added DVDS (597 $\mu \mathrm{L}$, $2.6 \mathrm{mmol}, 1.3$ equiv) neat, via syringe. The argon adapter was again removed, $\mathrm{KOSiEt}_{3}(1.0$ $\mathrm{g}, 6.0 \mathrm{mmol}, 3.0$ equiv) was quickly added, and the argon adapter was replaced. An additional $2 \mathrm{~mL}$ of DMF was then added via syringe, and the reaction was stirred for $4 \mathrm{~h}$. After the reaction was determined to be complete by GC analysis, the mixture was cooled to rt, then was 
partitioned between EtOAc $(50 \mathrm{~mL})$ and water $(50 \mathrm{~mL})$. The aqueous layer was extracted with EtOAc $(50 \mathrm{~mL})$, and the organic layers were washed with water $(50 \mathrm{~mL})$. The organic phases were combined, dried over $\mathrm{Na}_{2} \mathrm{SO}_{4}$ sulfate, then were filtered and concentrated in vacuo ( 25 ${ }^{\circ} \mathrm{C}, 50 \mathrm{~mm} \mathrm{Hg}$ ). Purification of the crude product by column chromatography (silica gel; 30 $\mathrm{mm} \times 120 \mathrm{~mm}$; pentane/EtOAc; 20/1) afforded $303 \mathrm{mg}$ (82\%) of 14i as a white solid. Data for 14i ${ }^{27 b}$ : mp: $85-86{ }^{\circ} \mathrm{C}$; ${ }^{1} \mathrm{H}$ NMR: $\left(500 \mathrm{MHz}, \mathrm{CDCl}_{3}\right)$ 7.72-7.69 (m, $\left.3 \mathrm{H}\right), 7.61(\mathrm{dd}, J=8.6$ and 1.5, $1 \mathrm{H}), 7.14-7.11(\mathrm{~m}, 2 \mathrm{H}), 6.85(\mathrm{dd}, J=17.6$ and $10.9,1 \mathrm{H}), 5.82(\mathrm{~d}, J=17.6,1 \mathrm{H})$, $5.28(\mathrm{~d}, J=10.9,1 \mathrm{H}), 3.92(\mathrm{~s}, 3 \mathrm{H}) ;{ }^{13} \mathrm{C}$ NMR: $\left(125 \mathrm{MHz},\left(\mathrm{CDCl}_{3}\right)\right.$ 158.0, 137.2, 134.6, 133.2, 129.8, 129.2, 127.2, 126.4, 124.0, 119.2, 113.3. IR: 3088, 3066, 2960, 2939, 2908, 2839, 1626, 1597, 1507, 1480, 1452, 1404, 1389, 1339, 1267, 1239, 1197, 1178, 1164, 1032, 994, 964, 896, 858, 826, 738; MS: (EI, 70eV) 184, 169, 141, 139, 115; $R_{f} 0.32$ (pentane/Et $2 \mathrm{O}, 20: 1$ ).

\section{Acknowledgments}

We are grateful to the National Institutes of Health for generous financial support. (R01 GM63167) and JohnsonMatthey for the gift of $\mathrm{Pd}_{2}(\mathrm{dba})_{3}$. C.R.B. thanks Abbott Laboratories for a fellowship, and the University of Illinois for a Seemon H. Pines Graduate Fellowship.

\section{References}

(1). (a) Agbossou F, Carpentier J-F. Chem. Rev 1995;95:2485-2506. (b) Crudden CM, Hleba YB, Chen AC. J. Am. Chem. Soc 2004;126:9200-9201. [PubMed: 15281807] (c) RajanBabu TV, Nomura N, Jin J, Nandi M, Park H, Sun X. J. Org. Chem 2003;68:8431-8446. [PubMed: 14575468]

(2). (a) Scheirs, J.; Priddy, DB., editors. Modern Styrenic Polymers: Polystyrenes and Styrenic Copolymers. John Wiley; Chichester: 2003. (b) Hirao A, Loykulnant S, Ishizone T. Prog. Polym. Sci 2002;27:1925-1982. (c) Schellenberg J, Tomotsu N. Prog. Polym. Sci 2002;27:1925-1982.

(3). Dieguez M, Pamies O, Claver C. Tetrahedron: Asymmetry 2004;15:2113-2122.

(4). (a) Hii KK. Pure App. Chem 2006;78:341-349. (b) Hultzsch KC. Org. Bio. Chem 2005;3:18191824.

(5). (a) Johnson, RA.; Sharpless, KB. Catalytic Asymmetric Synthesis. Vol. Second Edition. Ojima, I., editor. Wiley-VCH; Weinheim: 2000. Chapter 6D(b) Markó, I.; Svendsen, JS. Comprehensive Asymmetric Catalysis. Jacobsen, EN.; Pfaltz, A.; Yamamoto, H., editors. Vol. II. Springer Verlag; Heidelberg: 1999. Chapter 20

(6). (a) Xia Q-H, Ge H-Q, Ye C-P, Liu Z-M, Su K-X. Chem. Rev 2005;105:1603-1662. [PubMed: 15884785](b) Katsuki, T. Catalytic Asymmetric Synthesis. Ojima, I., editor. Vol. 2. VCH; New York: 2000. p. 287-326.(c) Jacobsen, EN. Catalytic Asymmetric Synthesis. Ojima, I., editor. Vol. 1. VCH; New York: 1993. p. 159-202.

(7). (a) Kotov V, Scarborough CC, Stahl SS. Inorg. Chem 2007;46:1910-1923. [PubMed: 17348722] (b) Wolfe JP. Eur. J. Org. Chem 2007:571-582.

(8). (a) Grubbs, RH. Tetrahedron. Vol. 60. 2004. p. 7117-7140.(b) Grubbs, RH., editor. Handbook of Metathesis. Wiley-VCH; Weinheim: 2003.

(9). Emerson WS. Chem. Rev 1949;49:347-383.

(10). (a) Heck RF. Org. React 1982;27:345-390. (b) Bhanage BM, Fujita S-F, Yoshida T, Sano Y, Arai M. Tetrahedron Lett 2003;44:3505-3507.

(11). (a) Peyroux E, Berthiol F, Doucet H, Santelli M. Eur. J. Org. Chem 2004:1075-1082. (b) Stewart SK, Whiting A. J. Organomet. Chem 1994;482:293-300. (c) Lightfoot AP, Twiddle SJR, Whiting A. Synlett 2005:529-531. (d) Kerins F, O’Shea DF. J. Org. Chem 2002;67:4968-4971. [PubMed: 12098315] (e) Molander GA, Brown AR. J. Org. Chem 2006;71:9681-9686. [PubMed: 17168585] (f) Molander GA, Rivero MR. Org. Lett 2002;4:107-109. [PubMed: 11772102] (g) Darses S, Michaud G, Genet J-P. Eur. J. Org. Chem 1999:1875-1883.

(12). (a) Littke AF, Schwarz L, Fu GC. J. Am. Chem. Soc 2002;124:6343-6348. [PubMed: 12033863] (b) Grasa GA, Nolan SP. Org. Lett 2001;3:119-122. [PubMed: 11429852] (c) McKean DR, Parrinello G, Renaldo AF, Stille JK. J. Org. Chem 1987;52:422-424.

(13). Bumagin NA, Luzikova EV. J. Organomet. Chem 1997;532:271-273. 
(14). Vinylboronic acid pinacol ester, (Aldrich, catalog no. 63348): \$2757/mol (\$18/g), 2,4,6Trivinylcyclotriboroxane (Aldrich, catalog no. 637998): \$3822/mol (\$17/g), Potassium vinyltrifluoroborate (Aldrich, catalog no. 655228): \$2364/mol (\$18/g).

(15). The preparation of potassium vinyltrifluoroborate does not involve vinylboronic acid as an intermediate, although the vinylboronic acid dimethyl ester is used. For its preparation see ref $11 \mathrm{e}$.

(16). Farina V, Krishnamurthy V, Scott WJ. Org. React 1997;50:3-652.

(17). (a) National Institute of Occupational Health and Safety. U.S. Government Printing Office; Washington: 1976. Pub. No. 77-115(b) Lassiter, DV.; Stewart, JH. Patty's Toxicology. Vol. 5th Ed.. Bingham, E.; Cohrssen, B.; Powell, CH., editors. Vol. 2. Wiley; Hoboken: 2001. p. 576-598.

(18). Tributyl(vinyl)tin (Aldrich, catalog no. 271438): \$3032/mol.

(19). Lando VR, Monteiro AL. Org. Lett 2003;5:2891-2894. [PubMed: 12889901]

(20). Yoshida J, Tamao K, Yamamoto H, Kakui T, Uchida T, Kumada M. Organometallics 1982;1:542549.

(21). Cragg, ST. Patty's Toxicology. Bingham, E.; Cohrssen, B.; Powell, CH,., editors. Wiley; Hoboken: 2001. DOI: 10.1002/0471435139.tox.093

(22). (a) Denmark SE, Yang S-M. Org. Lett 2001;3:1749-1752. [PubMed: 11405702] (b) Denmark SE, Yang S-M. J. Am. Chem. Soc 2002;124:2102-2103. [PubMed: 11878949] (c) Denmark SE, Yang S-M. J. Am. Chem. Soc 2002;124:15196-15197. [PubMed: 12487594] (d) Denmark SE, Yang SM. Tetrahedron 2004;60:9695-9708.

(23). Denmark SE, Kobayashi T. J. Org. Chem 2003;68:5153-5159. [PubMed: 12816470]

(24). Denmark SE, Liu JH-C. J. Am. Chem. Soc 2007;129:3737-3744. [PubMed: 17335205]

(25). Hosomi A, Kohra S, Tominga Y. Chem. Pharm. Bull. Jpn 1988;36:4622-4625.

(26). Hatanaka Y, Hiyama T. J. Org. Chem 1988;53:918-920.

(27). (a) Li J-H, Deng C-L, Xie YX. Synthesis 2006:969-974. (b) Alacid, E.1; Najera, C. Adv. Syn. Cat 2006;348:2085-2091.

(28). Denmark SE, Sweis RF. Acc. Chem. Res 2002;35:835-846. [PubMed: 12379136]

(29). Tamao K, Kobayashi K, Ito Y. Tetrahedron Lett 1989;30:6051-6054.

(30). (a) Denmark SE, Ober MH. Adv. Syn. Cat 2004;346:1703-1714. (b) Denmark SE, Neuville L. Org. Lett 2000;2:3221-3224. [PubMed: 11009386]

(31). Denmark SE, Sweis RF. J. Am. Chem. Soc 2001;123:6439-6440. [PubMed: 11427080]

(32). Denmark SE, Baird JD. Chem. Eur. J 2006;12:4954-4963.

(33). (a) Denmark SE, Kallemeyn JM. J. Am. Chem. Soc 2006;128:15958-15959. [PubMed: 17165708] (b) Denmark SE, Baird JD. Org. Lett 2006;8:793-795. [PubMed: 16468769] (c) Denmark SE, Sweis RF. J. Am. Chem. Soc 2004;126:4876-4882. [PubMed: 15080692]

(34). Jeffery T. Tetrahedron Lett 1999;40:1673-1676.

(35). Denmark SE, Wang Z. Synthesis 2000:999-1003.

(36). Denmark SE, Sweis RF, Wehrli D. J. Am. Chem. Soc 2004;126:4865-4875. [PubMed: 15080691]

(37). Denmark SE, Wang Z. J. Organomet. Chem 2001;621:372-375.

(38). 1,3,5,7-Tetramethyl-1,3,5,7-tetravinylcyclotetrasiloxane (Gelest, catalog no. SIT7900.0): \$67/mol.

(39). Denmark SE, Butler CR. Org. Lett 2006;8:63-66. [PubMed: 16381568]

(40). For an example of DoE in an academic setting, as well as an excellent introduction to the application of Design of Experiment, see:Aggarwal VK, Staubitz AC, Owen M. Org. Proc. Res. Dev 2006;10:64-69.and references therein.

(41). For our first iteration, we employed a trial version of Fusion Pro developed by S-Matrix corporation

(42). To maintain a level of consistency, we operated under the assumption that each silicon atom would be able to transfer one vinyl group, regardless of the number of vinyl groups attached. Under this assumption, DMVSiOEt could transfer single vinyl donor, whereas HVDS and DVDS could transfer two vinyl units, and $\mathrm{D}_{4} \mathrm{~V}$, four vinyl units.

(43). See Supporting Information for design of reactor.

(44). Toluene was eliminated because of poor solubility of the reagent issues as well as direction from concurrent work of other members in the group. Additionally, only modest benefit accrued from 
the use of bulky, electron rich ligands and ligand $\mathbf{1 0}$ was eliminated from consideration because of cost. Moreover, the literature suggests that these Buchwald-type ligands are required not for crosscouplings of this type.

(45). The calculations and analysis for this and the third iteration used the commercially available Design Expert 7; StatEase, Inc. 2021 East Hennepin Ave., Suite 480, Minneapolis, MN, 55413, 2006.

(46). Denmark SE, Smith RC, Tymonko SA. Tetrahedron 2007;63:5730-5738.

(47). DVDS: \$52/mol, Gelest cat. \# SID4613; DMVSiOEt \$231/mol, Gelest cat \# SIV9070; HVDS: $\$ 1,320 / \mathrm{mol}$, Gelest cat. \# SIH6162

(48). See Supporting Information for experimental detail.

(49). Klapshina LG, Kurskii YA, Liepins E, Terman LM, Shaulova SV. Organomet. Chem. USSR 1991;4:16-18.

(50). For comparison, allylic alcohol has a pKa of 15.5 whereas ethanol has a pKa of 15.9,Ballinger P, Long FA. J. Am. Chem. Soc 1960;82:795-798.

(51). Relative bond dissociation energies: $\mathrm{C}_{6} \mathrm{H}_{5}-\mathrm{I}: 65 \mathrm{kcal} / \mathrm{mol}, \mathrm{C}_{6} \mathrm{H}_{5}-\mathrm{Br}: 81 \mathrm{kcal} / \mathrm{mol}$.Grushin VV, Alper H. Chem. Rev 1994;94:1047-1062.

(52). The distribution of products was strikingly dependent on the source of the $\mathrm{KOSiMe}_{3}$. In reactions using $\mathrm{KOSiMe}_{3}$ purchased from Gelest (two distinct lots), the reduction of the aryl bromide to the corresponding arene was observed at elevated temperature both in the presence and absence of a ligand. Moreover, from a number of deuterium labeling experiments it was determined the hydride was coming from the $\mathrm{KOSiMe}_{3}$ obtained from Gelest. In reactions using $\mathrm{KOSiMe}_{3}$ obtained from Aldrich (two distinct lots), no such reduction was observed. To resolve this discrepancy, $\mathrm{KOSiMe}_{3}$ was prepared by the Gelest method and was found to be indistinguishable from the material obtained from Aldrich. Therefore, the use of $\mathrm{KOSiMe}_{3}$ from Aldrich for the vinylation of aryl bromides is recommended. The contaminant in the Gelest $\mathrm{KOSiMe}_{3}$ that is responsible for the reduction was never identified.

(53). (a) Wolfe JP, Singer RA, Yang BH, Buchwald SL. J. Am. Chem. Soc 1999;121:9550-9561. (b) Barder TE, Walker SD, Martinelli JR, Buchwald SL. J. Am. Chem. Soc 2005;127:4685-4696. [PubMed: 15796535]

(54). Indeed even in the absence of DVDS, 4-bromoanisole was converted to the aniline by C-N coupling when combined at $50{ }^{\circ} \mathrm{C}$ with $\mathrm{KOSiMe}_{3}$ and ligand $\mathbf{1 0}$.

(55). The low yield of $\mathbf{1 1}$ is attributable to the dimerization of $\mathbf{2}$.

(56). See Supporting Information for experimental detail.

(57). The ratio of $\mathrm{KOSiEt}_{3}$ to DVDS was chosen to keep the ratio of activator to available vinyl group consistent with the aryl iodide vinylation reactions. In the case of the iodides 3.5 equiv $\mathrm{KOSiMe}_{3}$ and 0.75 equiv DVDS were empirically identified from the DoE optimization. Because both vinyl groups of DVDS are available the ratio of activator to vinyl group is 3.5 to 1.5 (2.3 to 1). Under an original (but later disproven) assumption that only one dimethylvinylsilanolate was available under $\mathrm{KOSiEt}_{3}$ activation, we began with a 2.3 to 1 ratio of $\mathrm{KOSiEt}_{3}$ to DVDS (in practice 2.8 to 1.2).

(58). Denmark SE, Smith RC. Synlett 2006;18:2921-2928.

(59). Based upon this assumption, it is unclear why NMP performs so poorly in these reactions.

(60). Langanis ED, Chenard BL. Tetrahedron Lett 1984;25:5831-5834.

(61). Hirao A, Loykulnant S, Ishizone T. Prog. Poly. Sci 2002;27:1399-1471.

(62). Because the formation of dimethylamine was avoided by using $\mathrm{KOSiEt}_{3}$, there was no need to speculate on the rate of formation of $\mathbf{D}$ or its relevant equilibria.

(63). From the knowledge that independently prepared $\mathbf{1 1}$ couples with bromides at room temperature in the presence of $\mathbf{1 0}$, and the knowledge that $\mathbf{1 1}$ is generated rapidly at room temperature from DVDS and $\mathrm{KOSiMe}_{3}$, we can conclude that in-situ generated 11 cannot complete with $\mathrm{KOSiMe}_{3}$ and that exchange cannot take place.

(64). Echavarren AM, Stille JK. J. Am. Chem. Soc 1987;109:5478-5486. 
<smiles>C=C[W]</smiles><smiles>[R]c1ccccc1[X]</smiles>

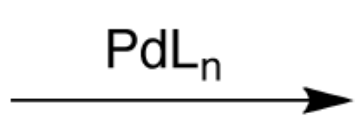<smiles>[R]c1ccccc1C=C</smiles>

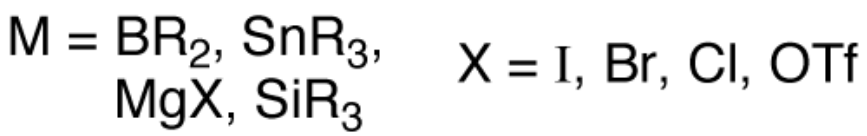

Scheme 1. 


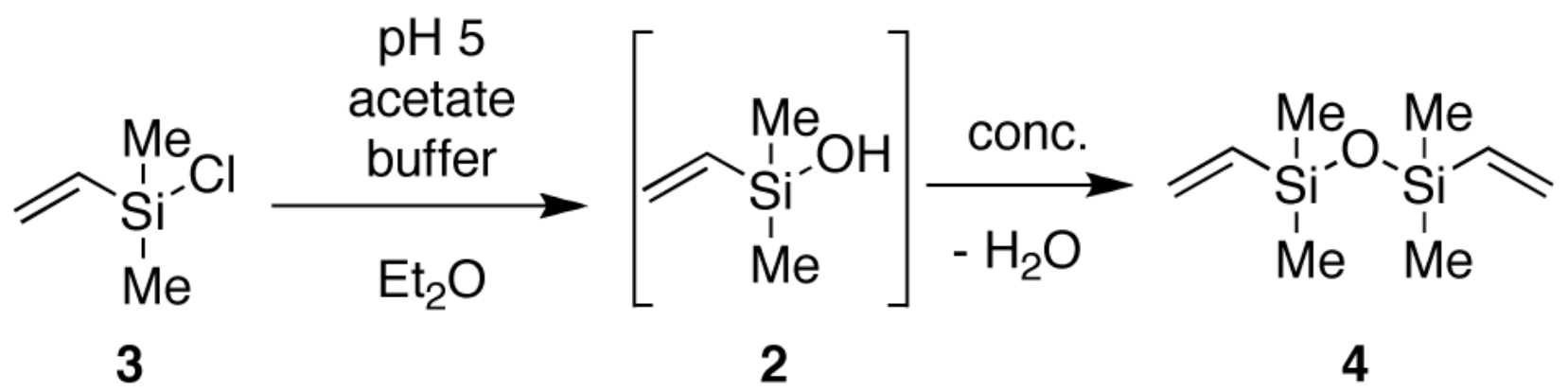

Scheme 2. 

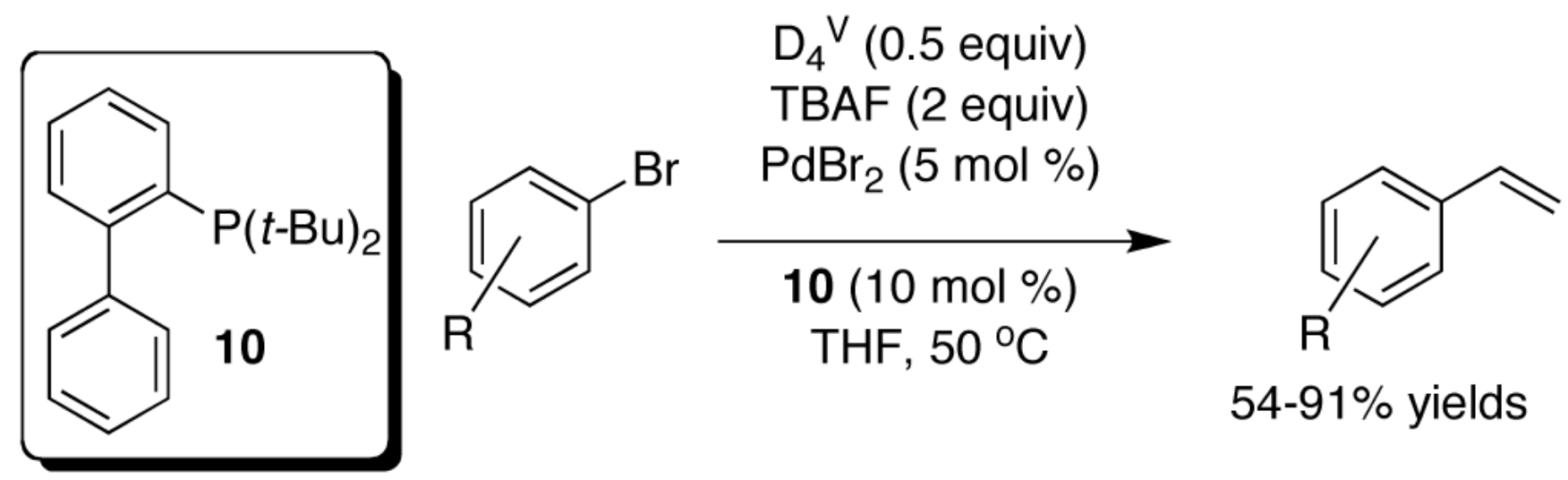

Scheme 3. 


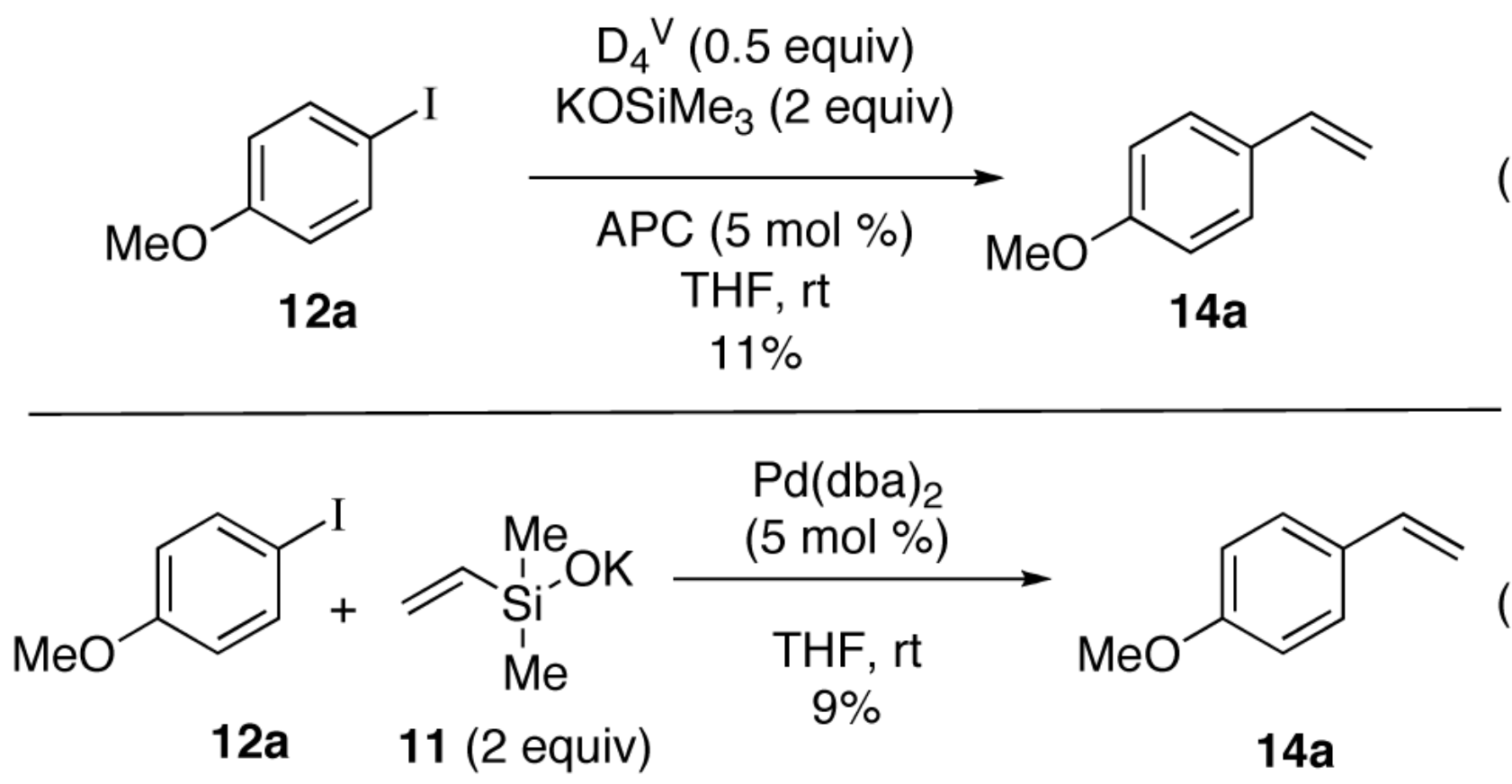

Scheme 4. 


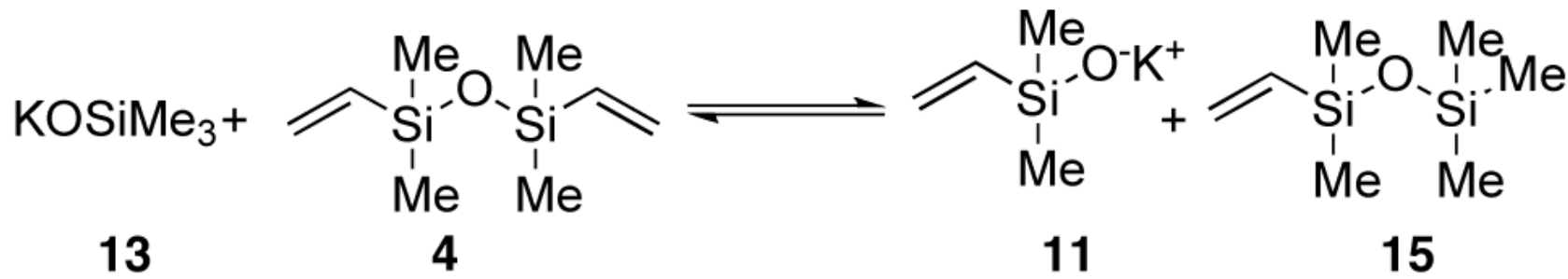

Scheme 5 . 
$\mathrm{KOSiMe}_{3}$ (4 equiv)

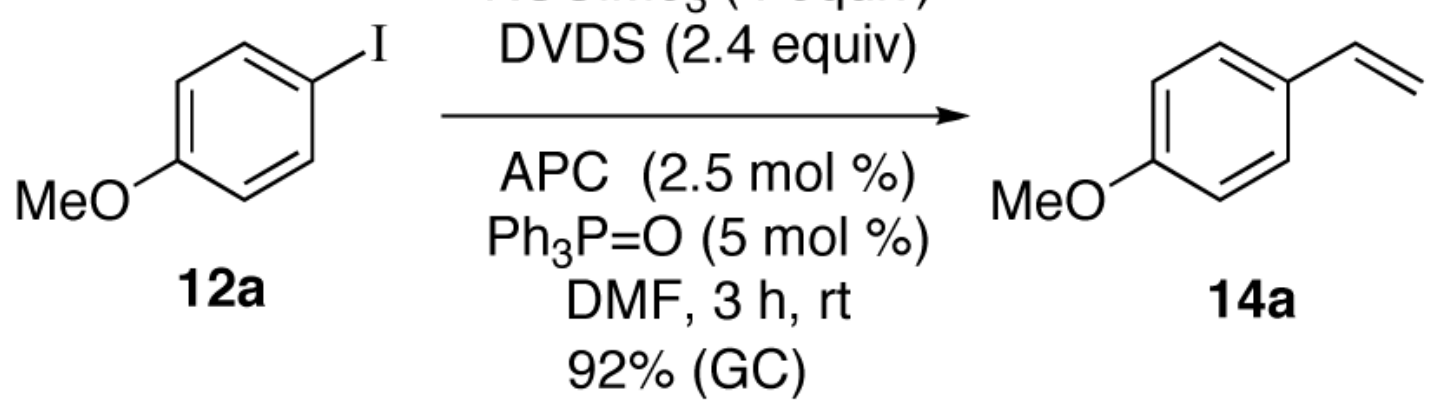

Scheme 6. 


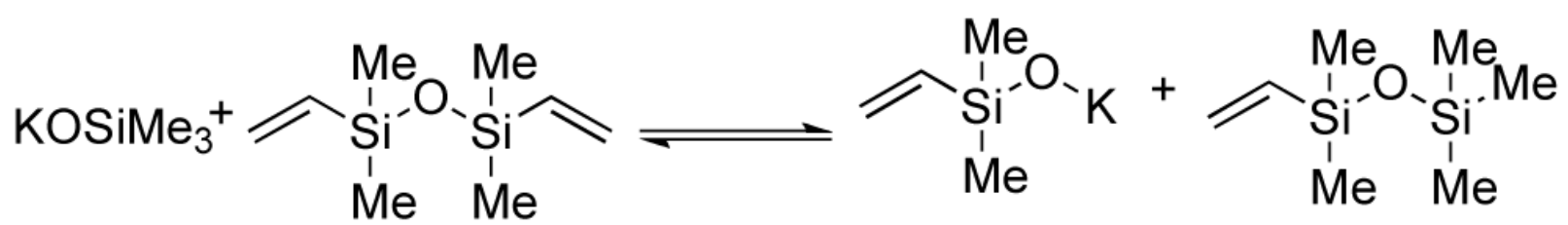

13

4

11

15

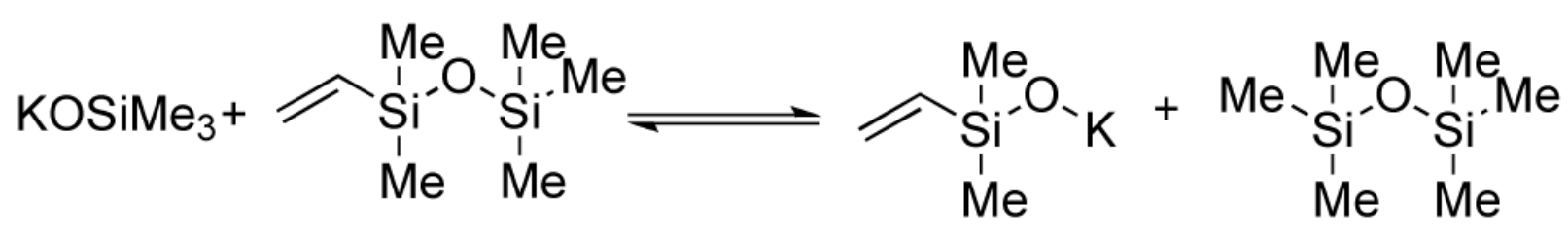

13

15

11

16

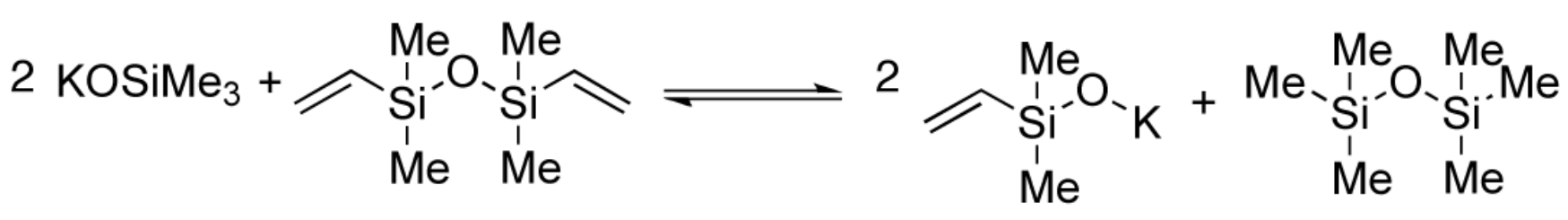

Scheme 7. 


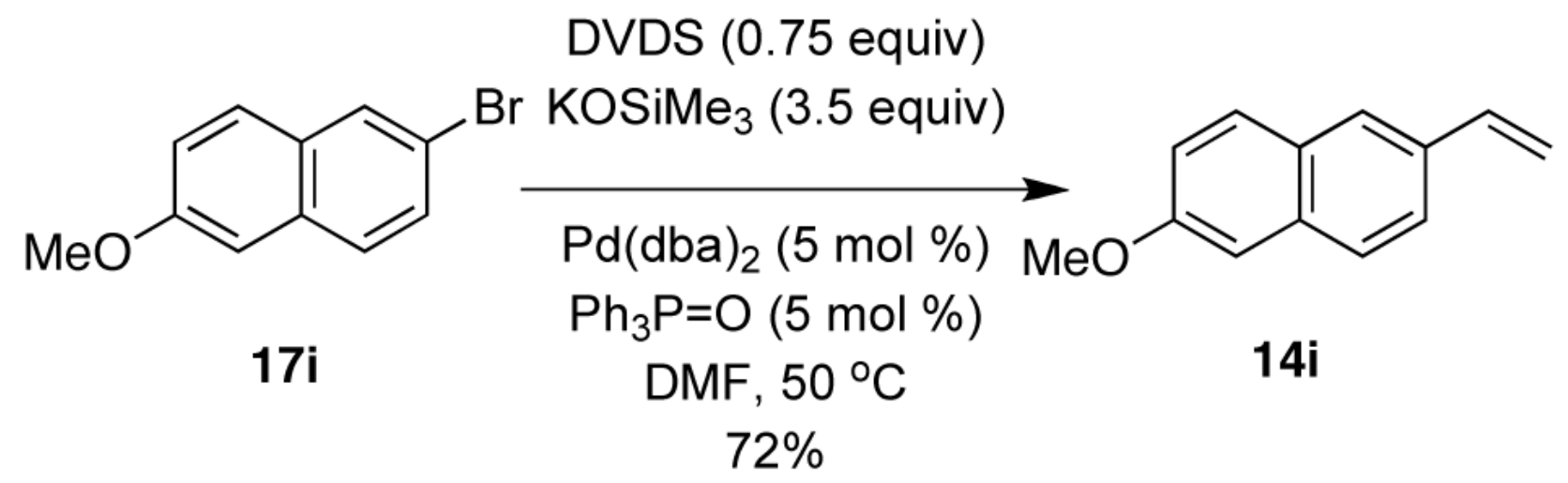

Scheme 8. 


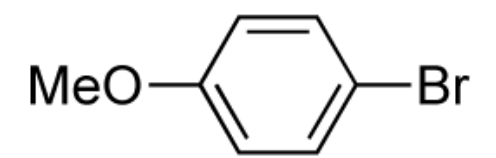

$17 a$

DVDS ( 0.75 equiv)

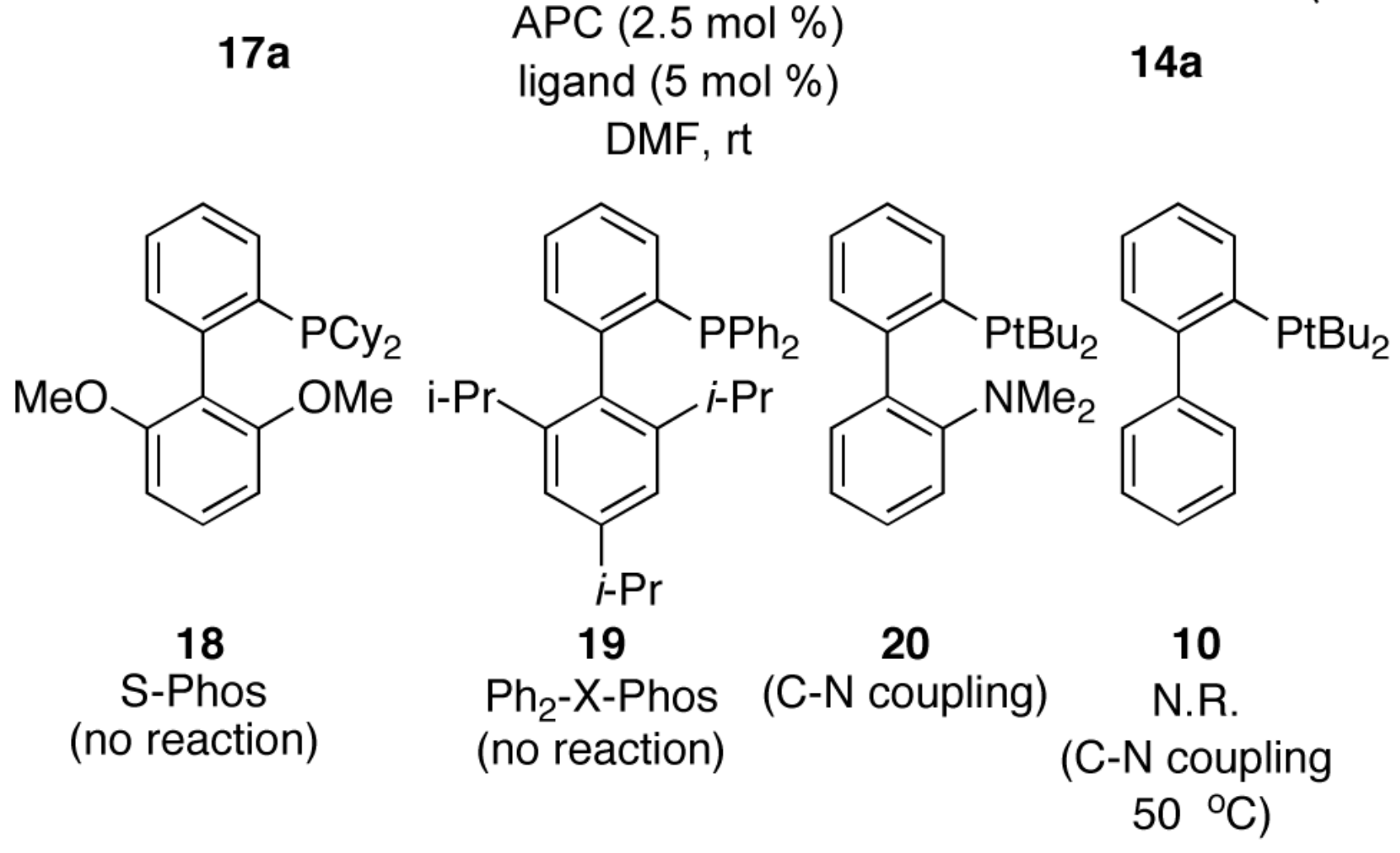

Scheme 9. 


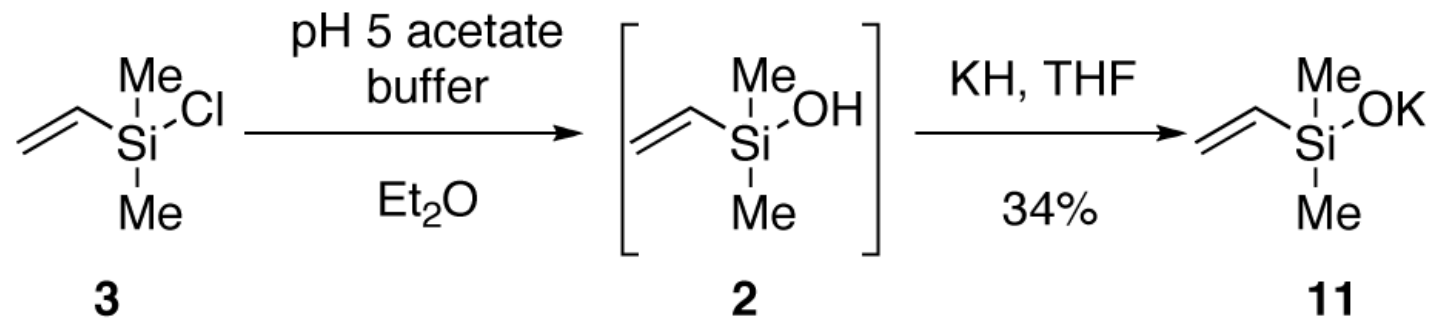

Scheme 10. 


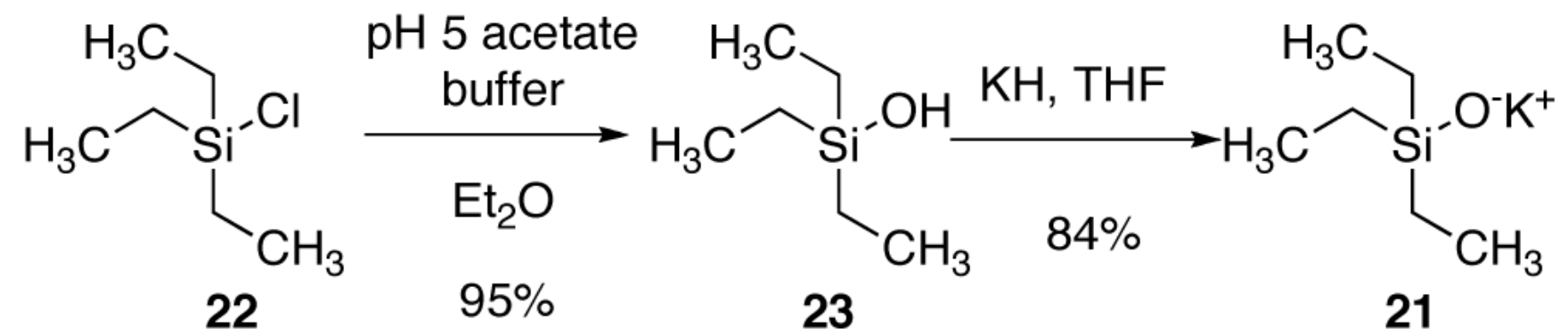

Scheme 11. 


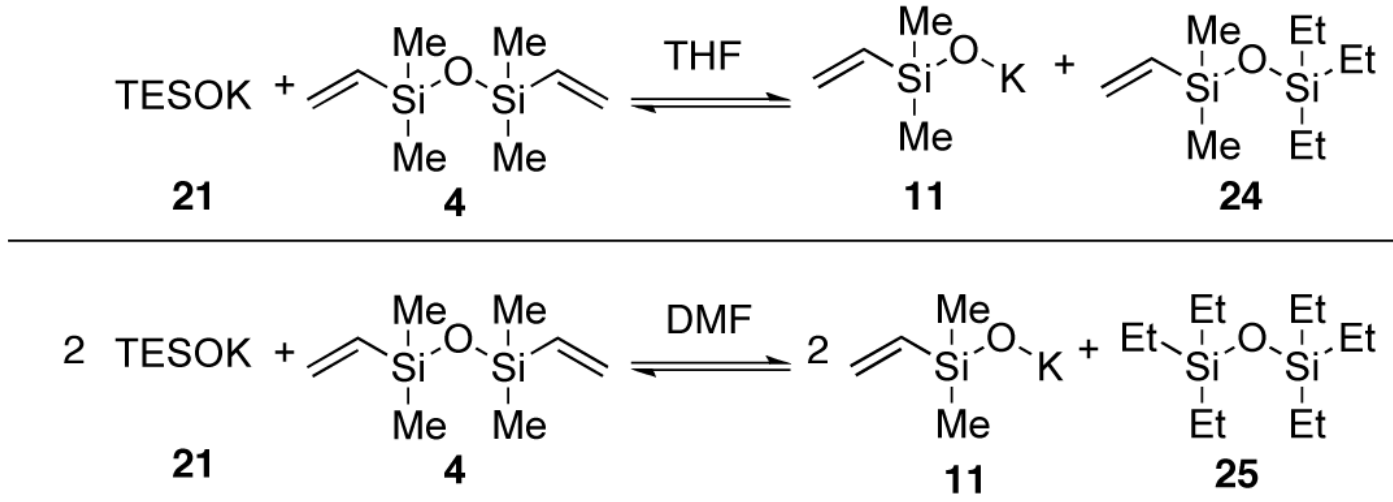

Scheme 12. 
DVDS (1.2 equiv)

$\mathrm{KOSiEt}_{3}$ (2.8 equiv)

$\mathrm{MeO}$

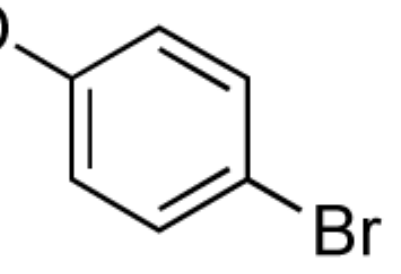

$17 a$
$\mathrm{MeO}$

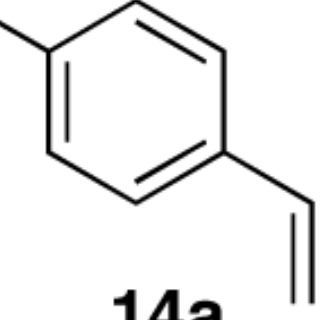

$14 a$

$63 \%$

Scheme 13. 
<smiles>Br[Te]c1ccccc1</smiles>

A

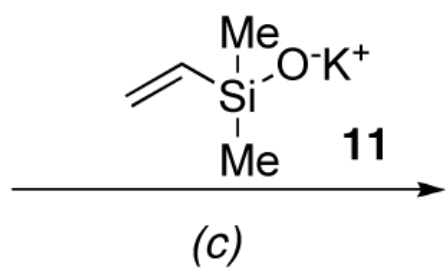

14

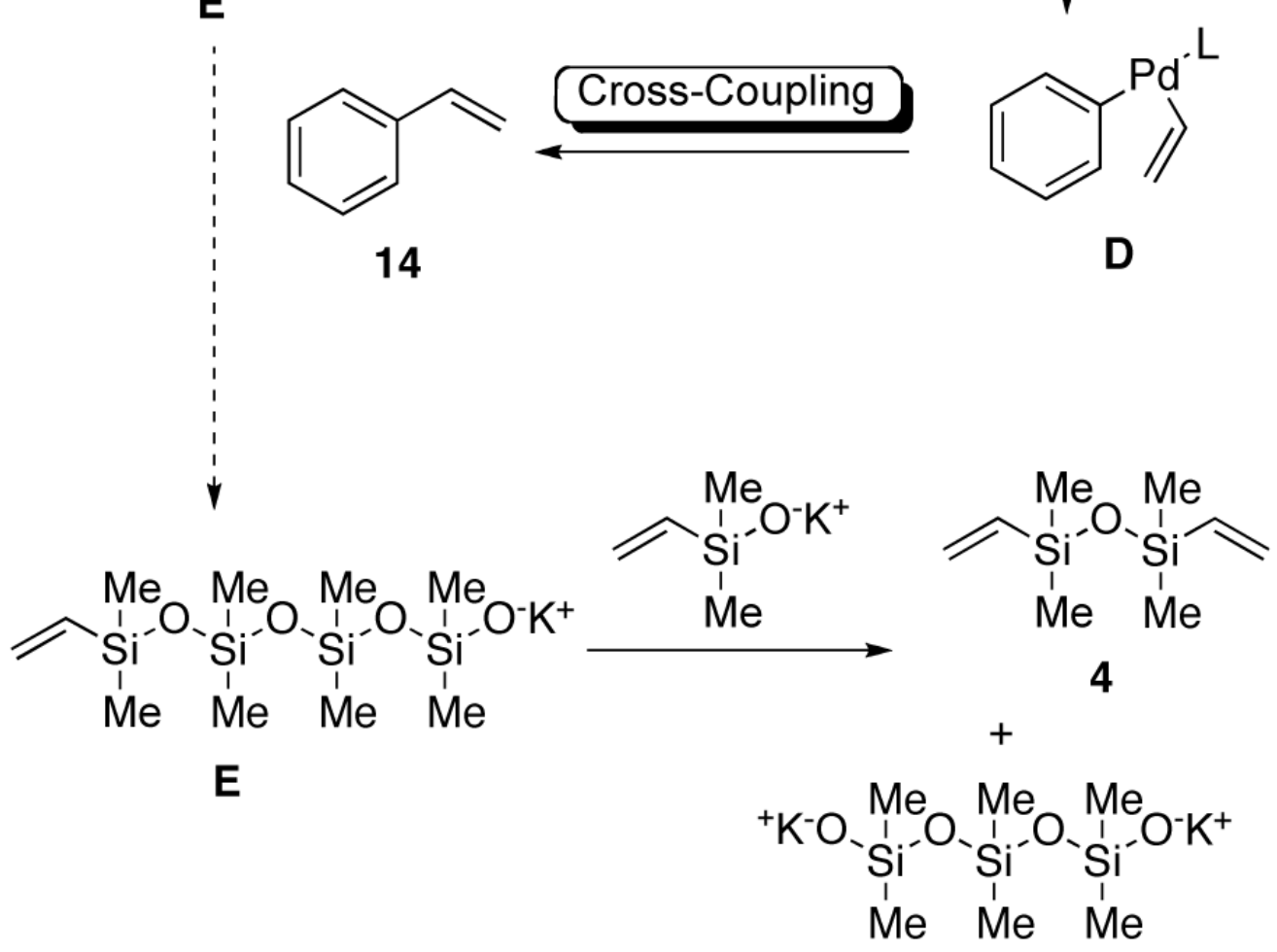<smiles>C=C[Si](C)(C)O[Te](I)c1ccccc1</smiles>

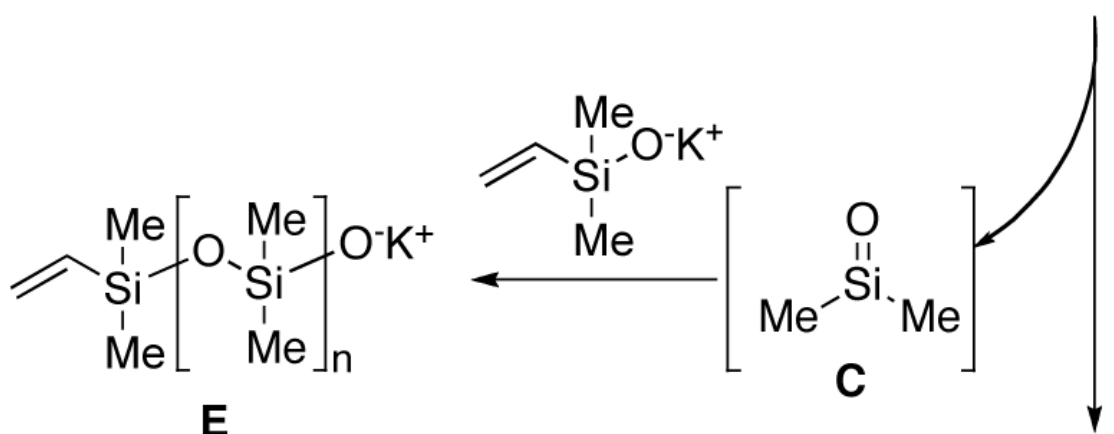

Scheme 14. 
<smiles>C/C=C\[Si](C)(C)O[Si](C)(C)C</smiles><smiles>CNC(=O)C=O</smiles>

(a)<smiles>[R][Si]([R])([R])O[Si](C)(C)C=C</smiles>

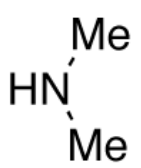

11

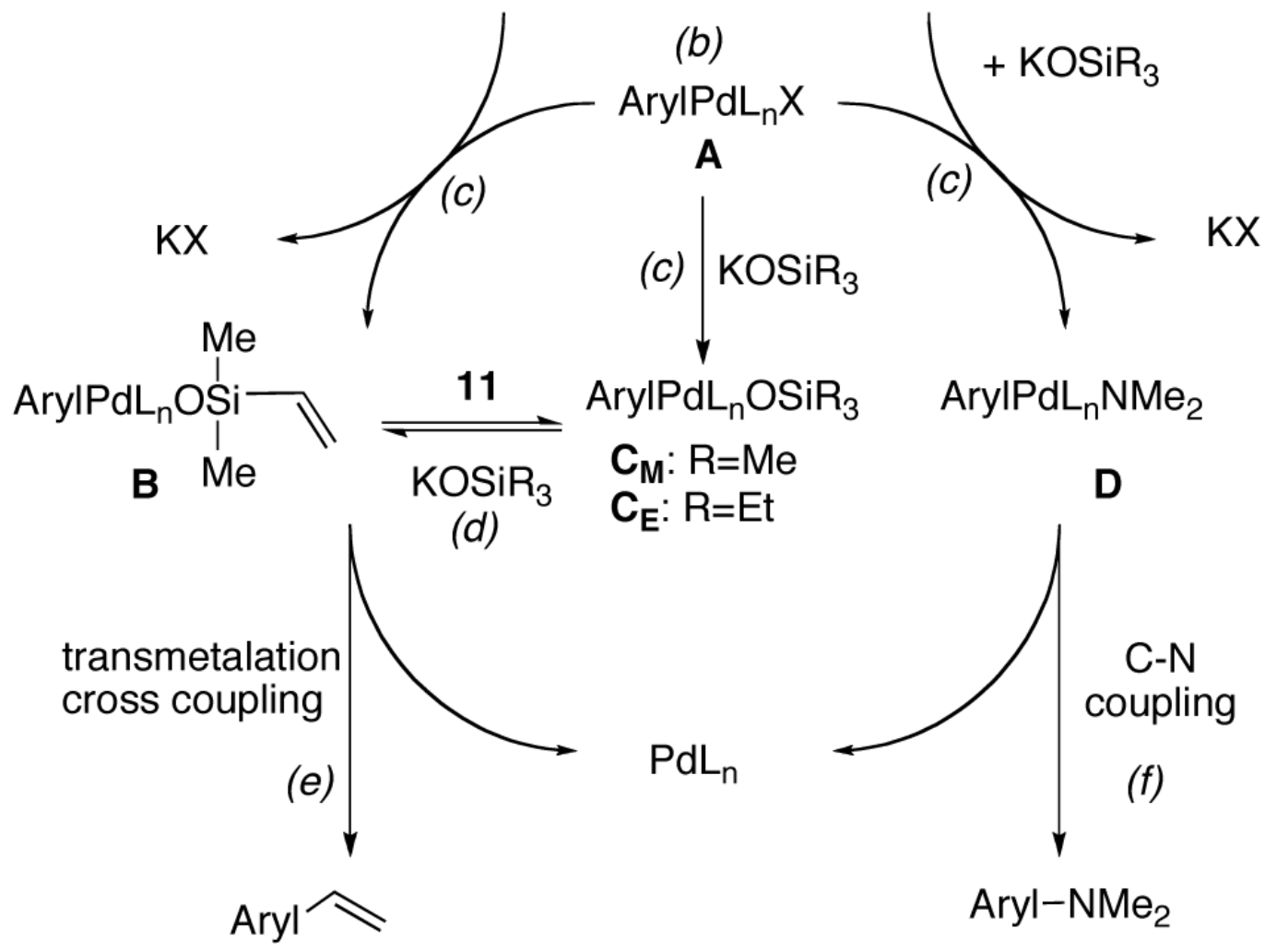

Scheme 15. 

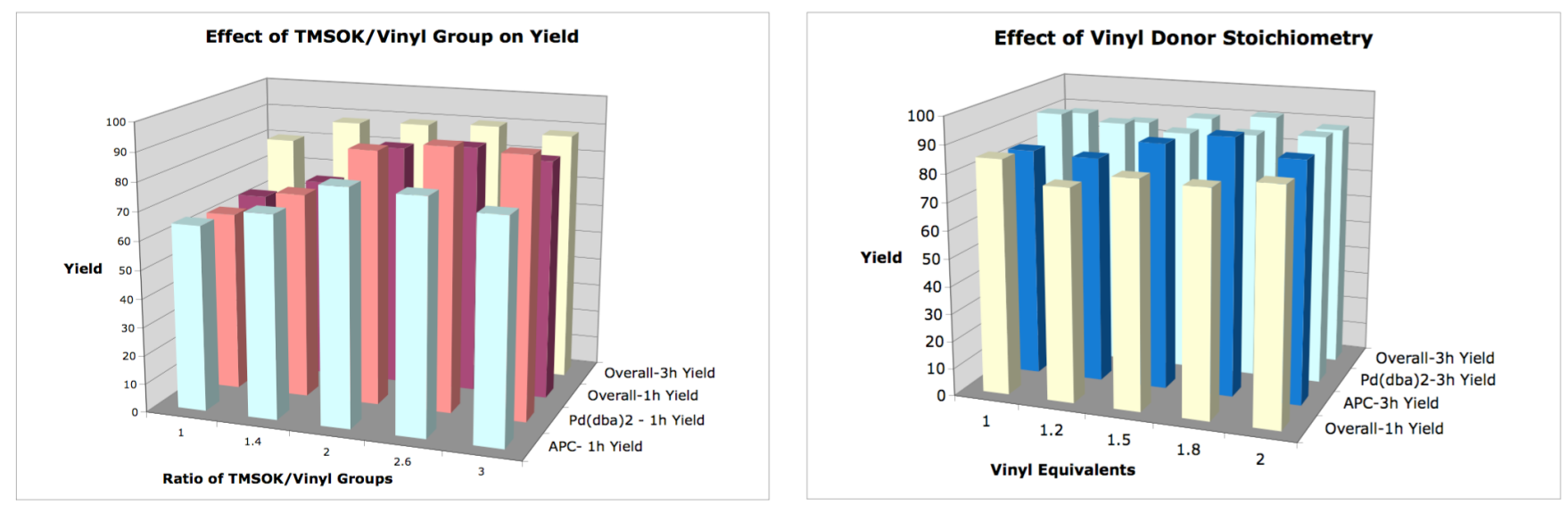

Figure 1.

Effect of vinyl donor and activator stoichiometry on yield from the third DoE 
$\underline{\operatorname{APC}(2.5 \%), \mathrm{Ph}_{3} \mathrm{P}=\mathrm{O}(5 \%)}$

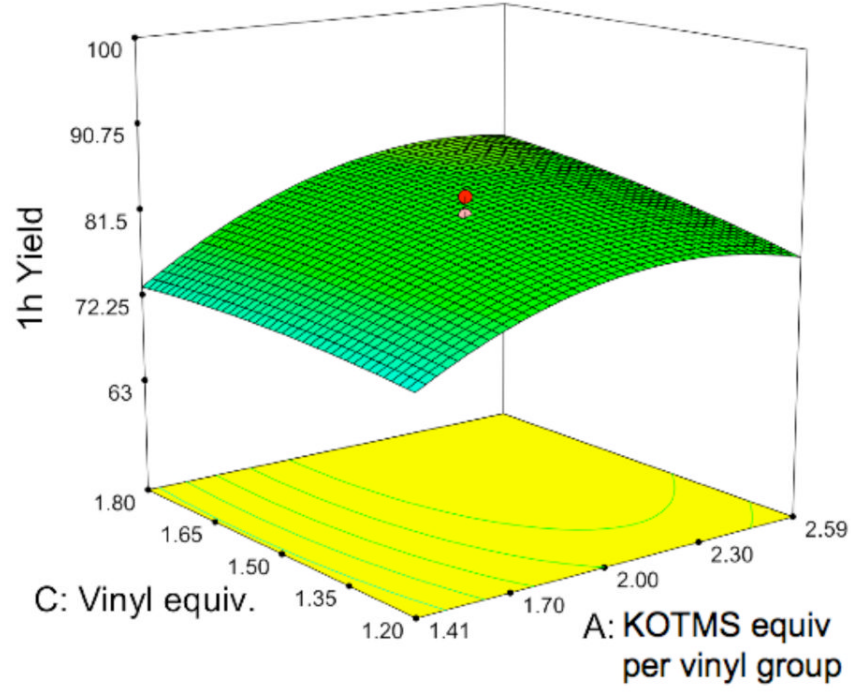

Figure 2.
$\underline{\mathrm{Pd}(\mathrm{dba})_{2}}{ }_{2}(5 \%), \mathrm{Ph}_{3} \mathrm{P}=\mathrm{O}(5 \%)$
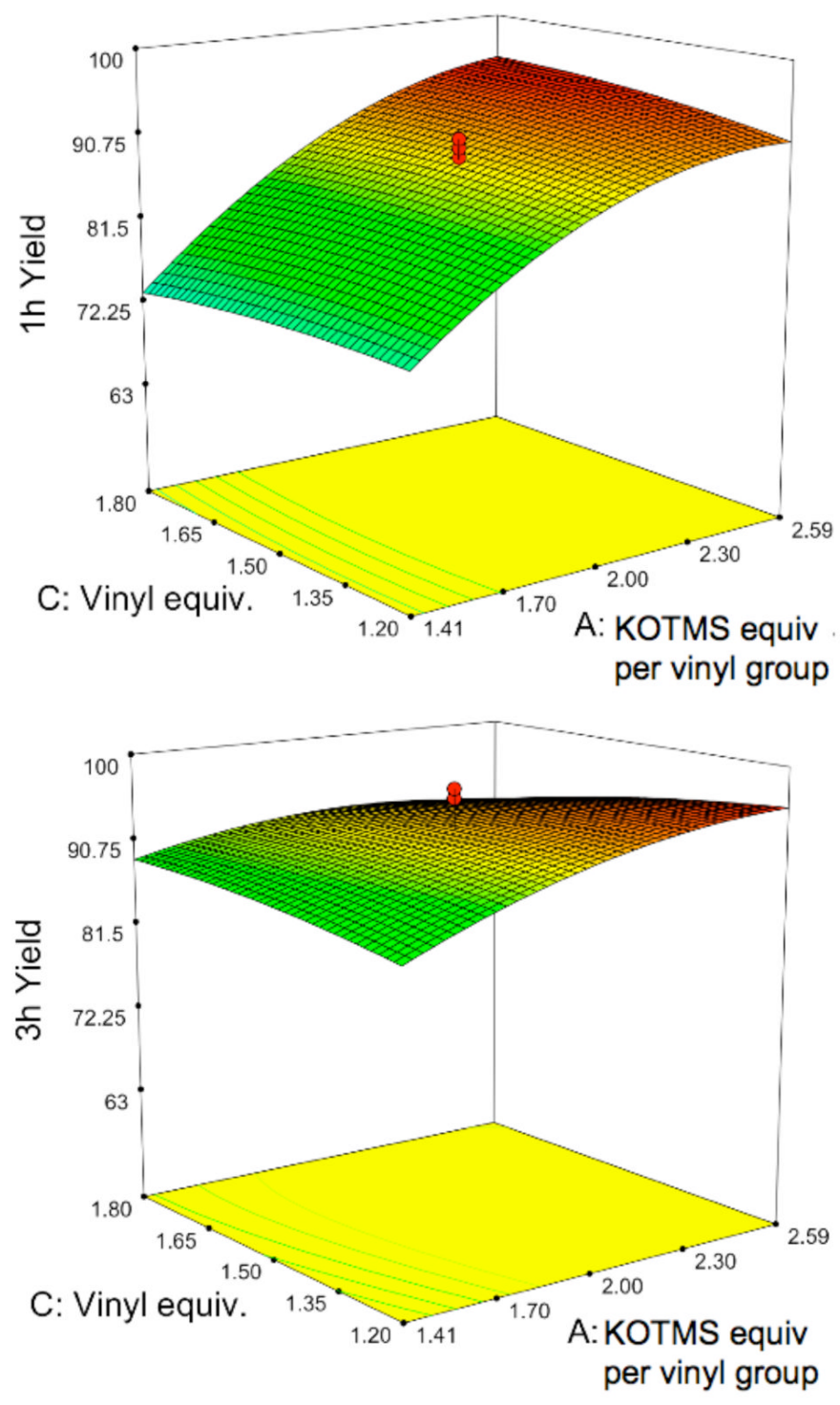

Three-Dimensional Surface Relationships Between TMSOK and Vinyl Equivalents 


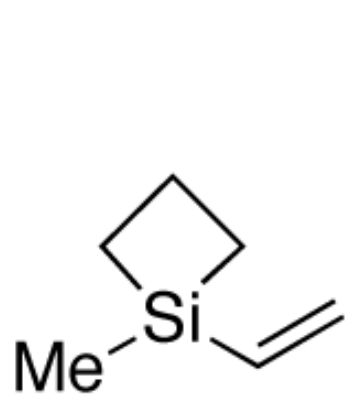

1

Chart 1.

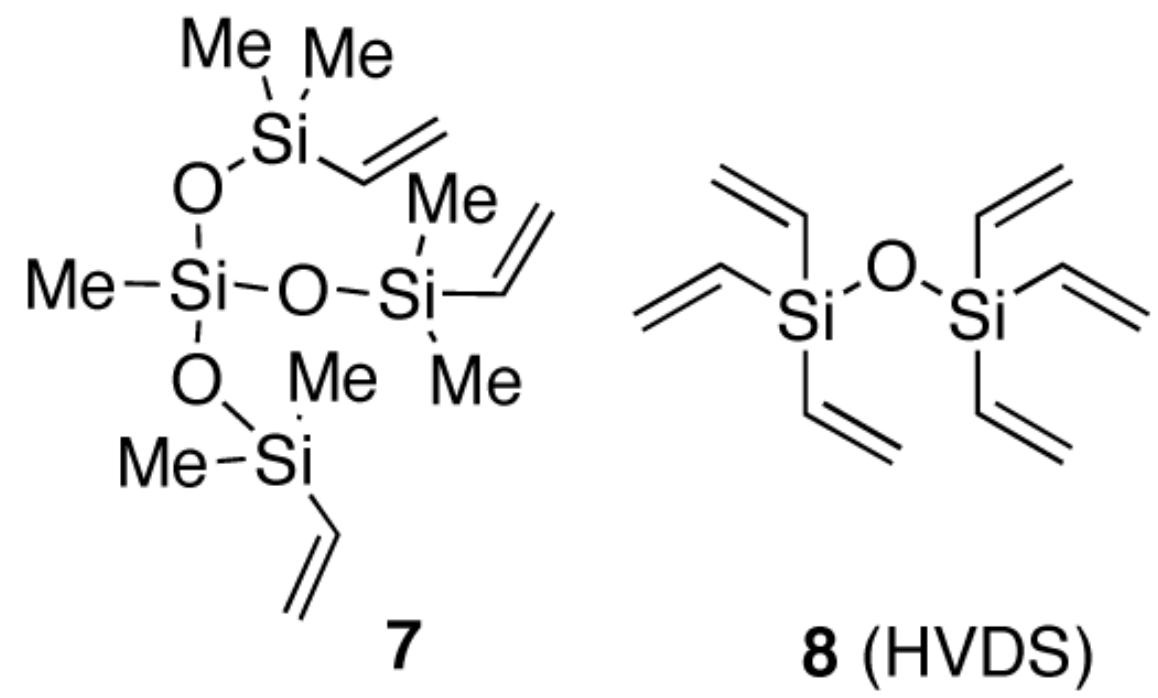

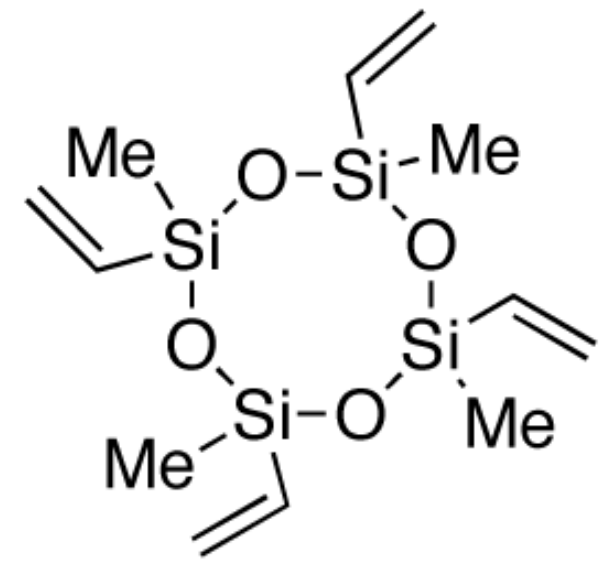

$5\left(D_{4} V^{V}\right)$

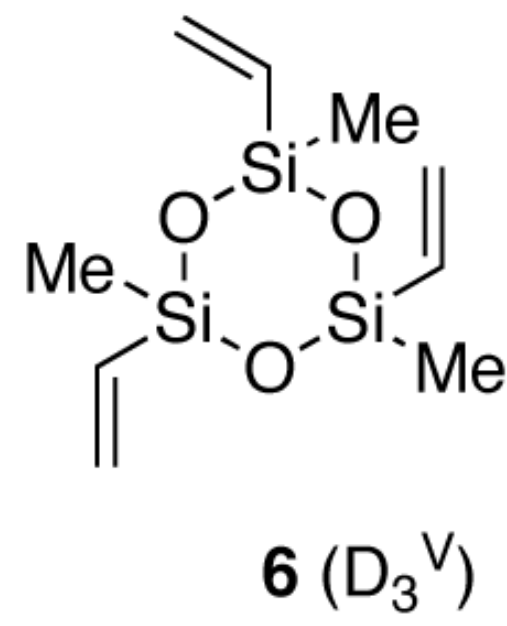

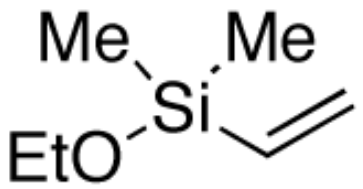

9 (DMVSOEt) 
<smiles>Brc1ccccn1</smiles><smiles>Nc1ccccc1Br</smiles>

26

27

28
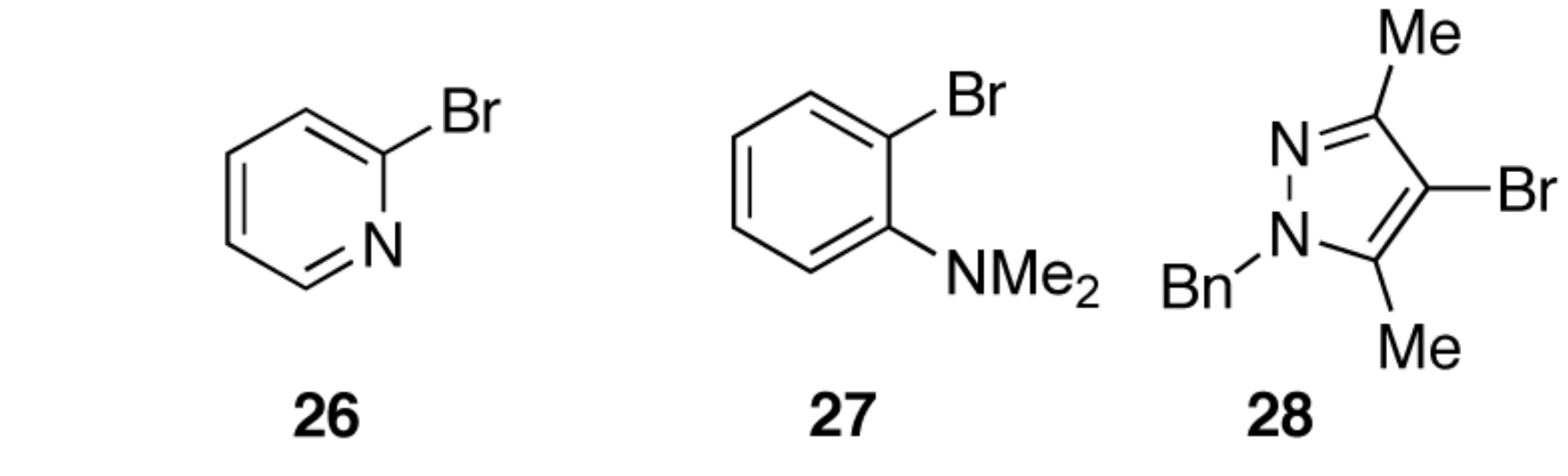

$\mathrm{Me}$<smiles>NC(=O)Nc1ccc(Br)cc1</smiles><smiles>CC(=O)c1ccc(Br)cc1</smiles>

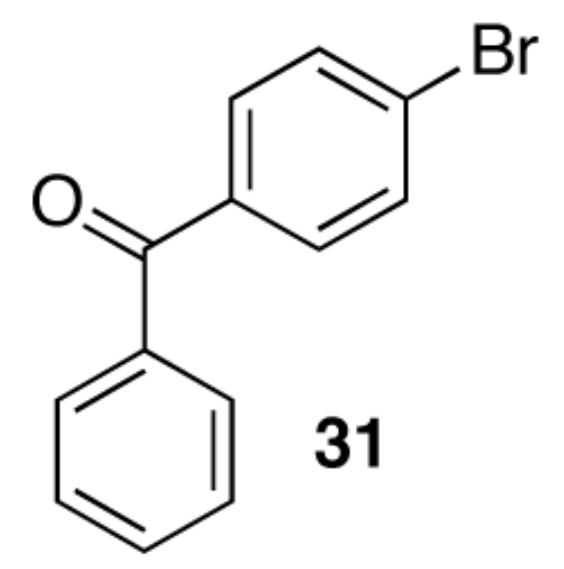<smiles>O=C(O)c1ccc(Br)cc1</smiles>

30

32

\section{Chart 2.}


Table 1

Vinylation using $\mathrm{KOSiMe}_{3}$ and $\operatorname{DVDS}^{a}$

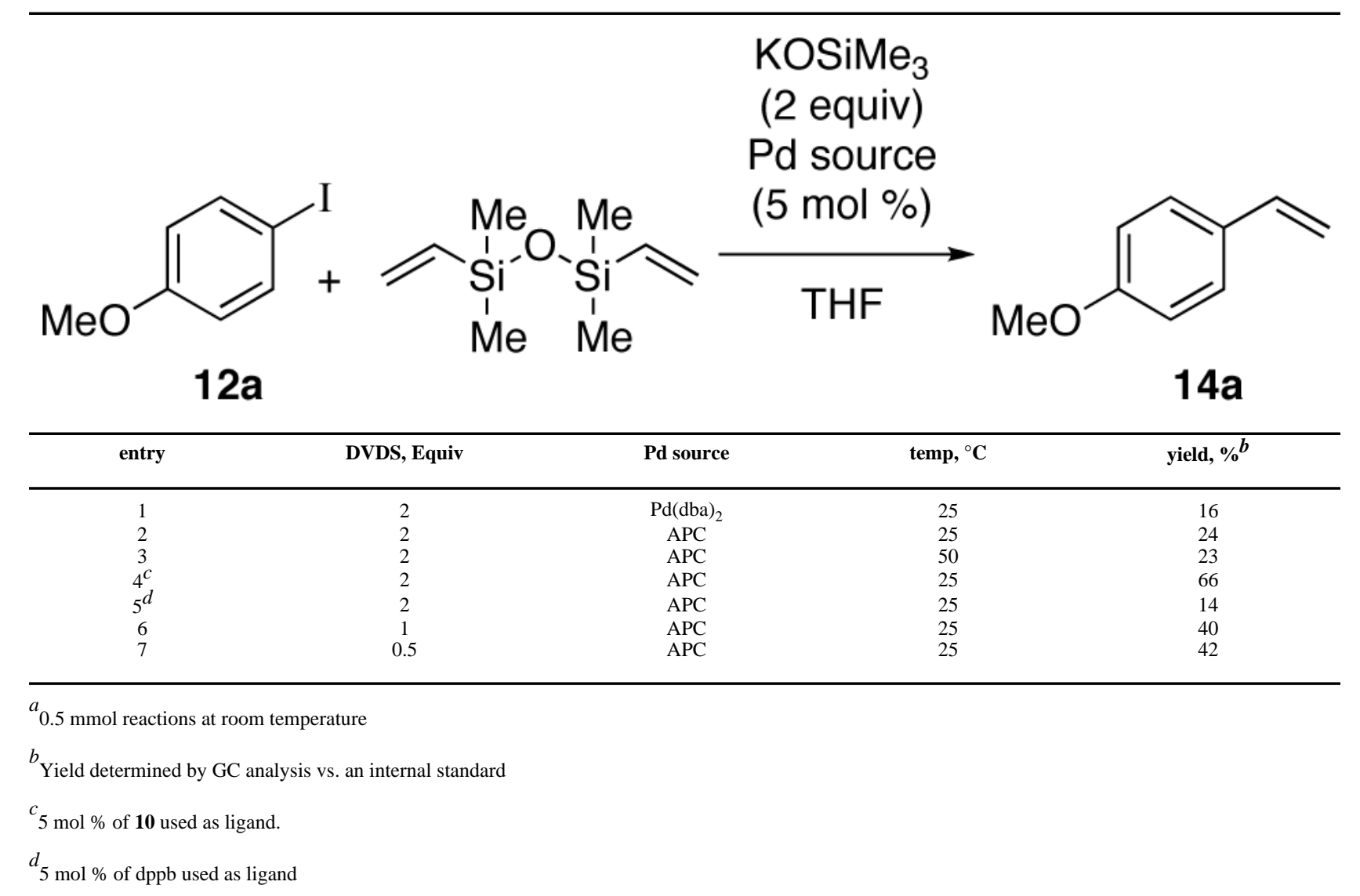




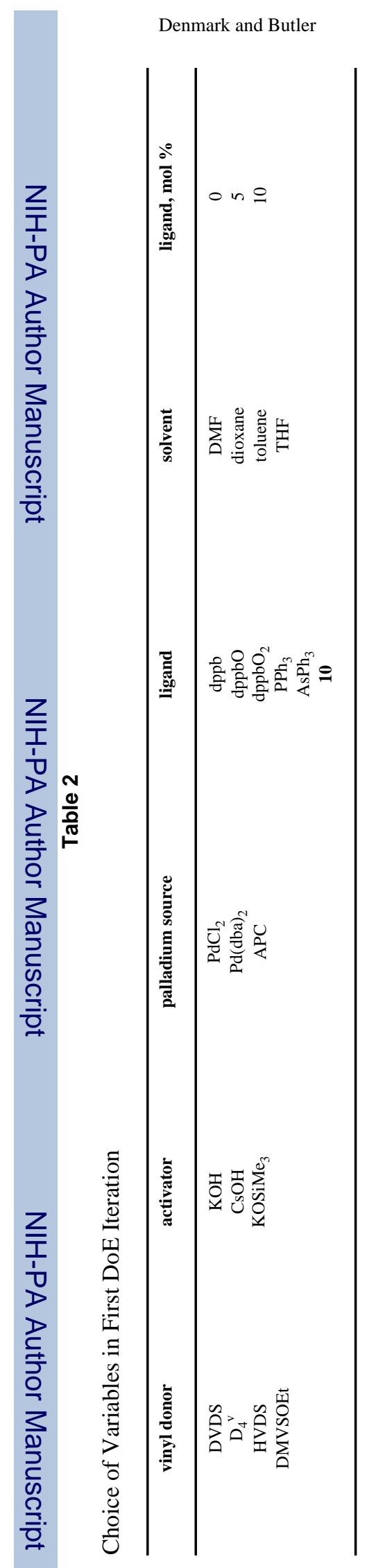

Page 39 


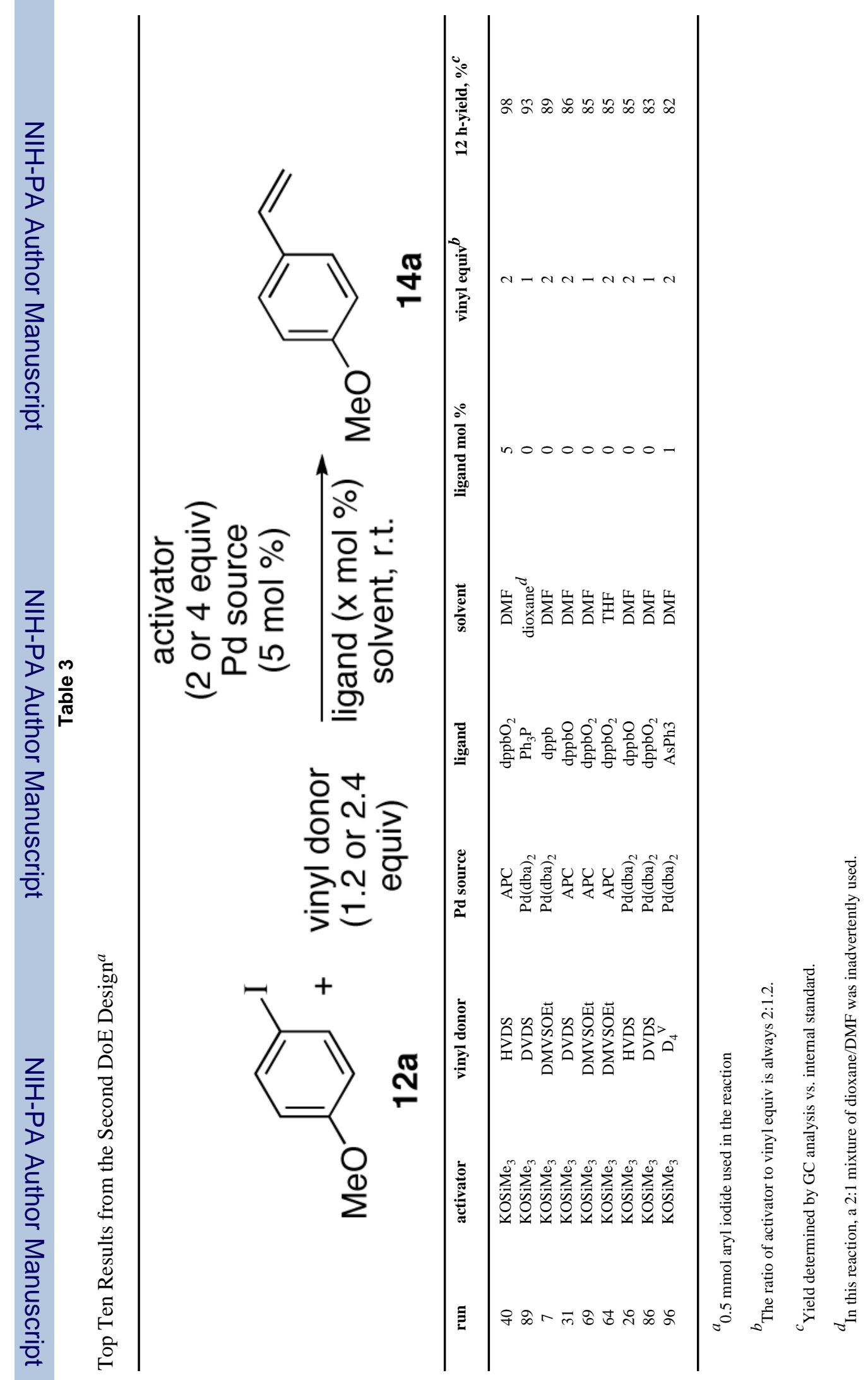


Table 4

Survey of Vinyl Donors

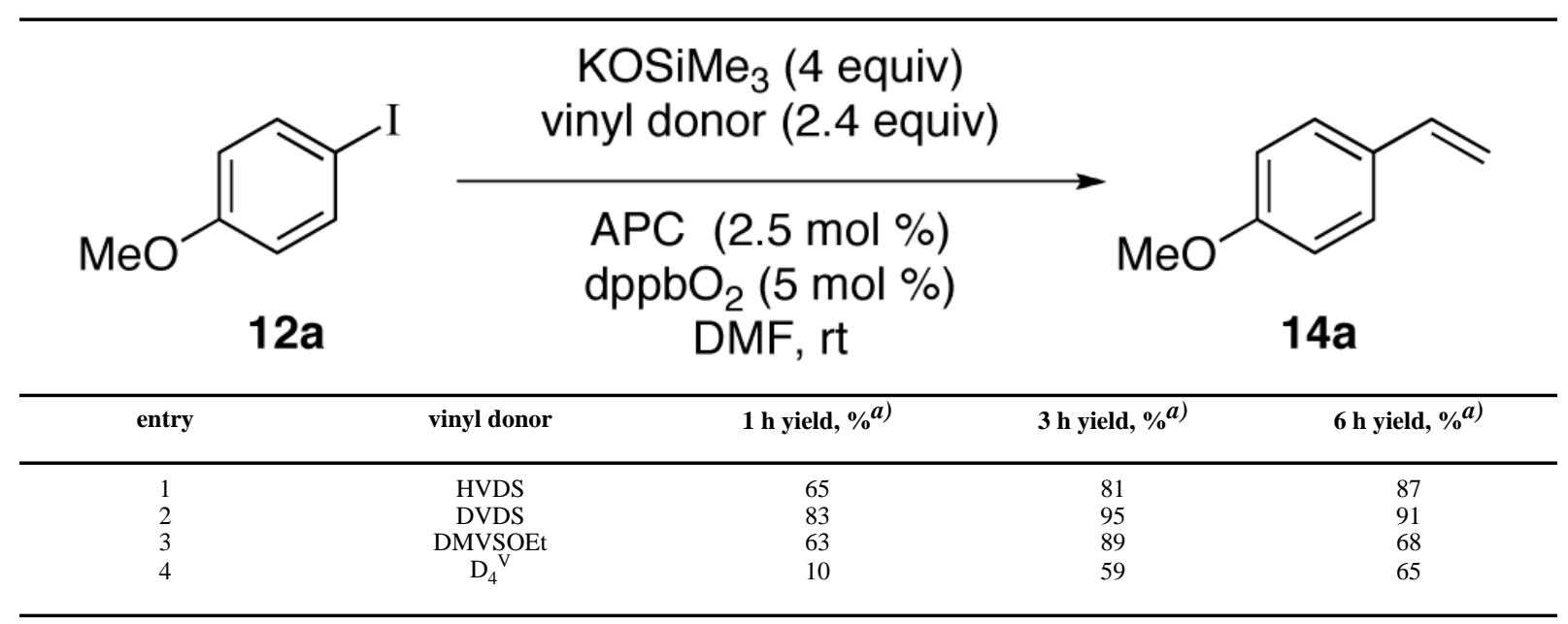

${ }^{a)}$ Determined by GC analysis vs. an internal standard. 


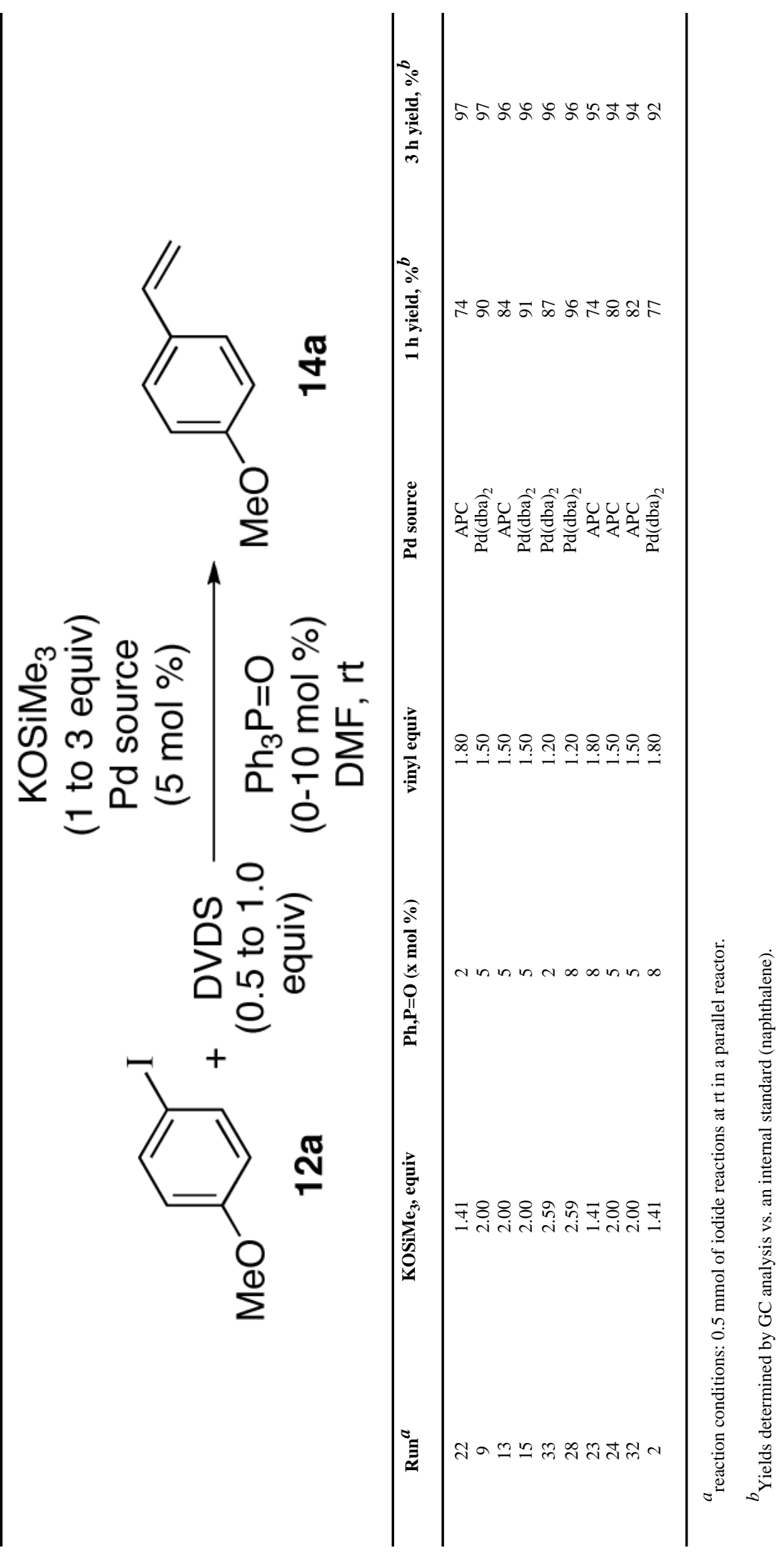




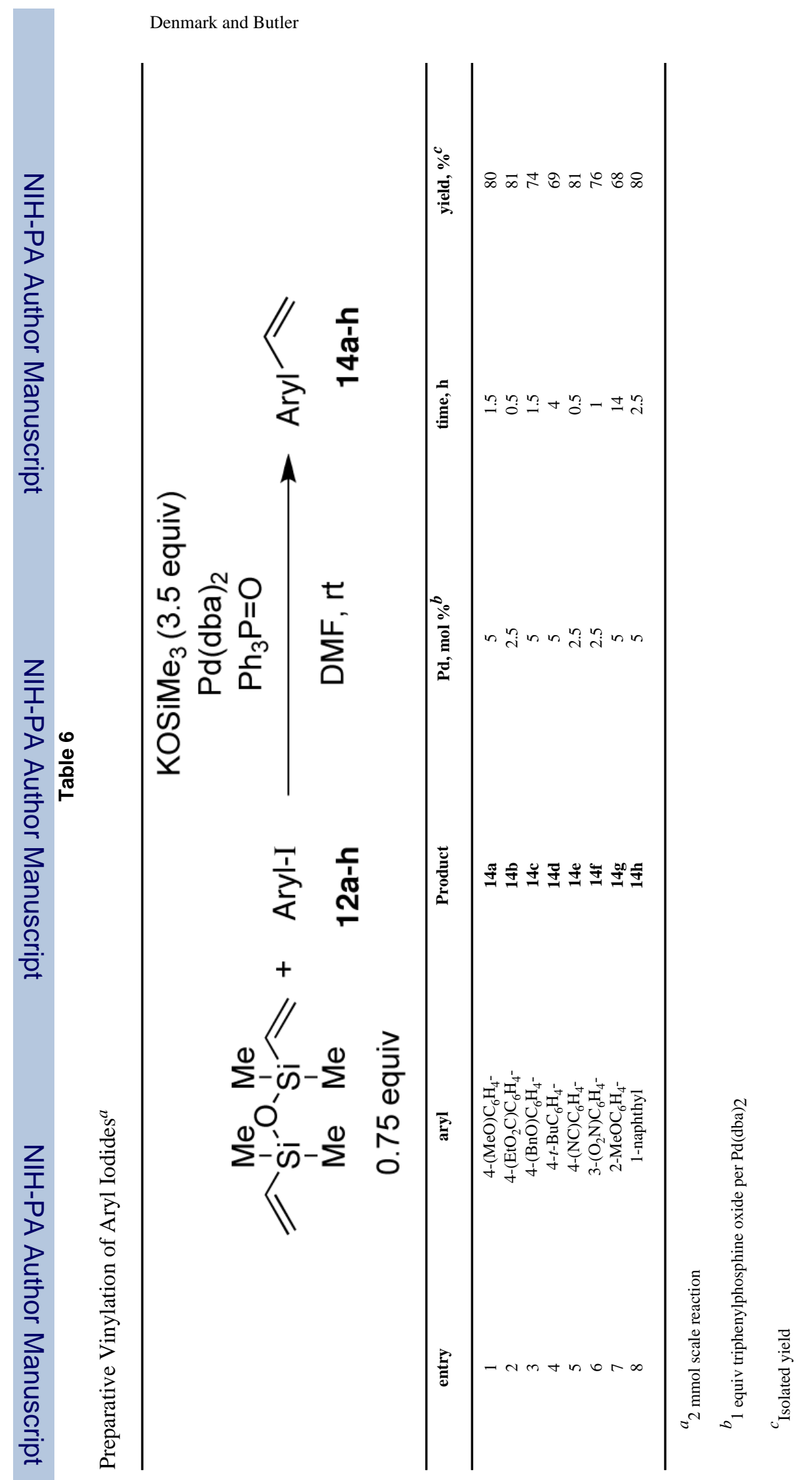

J Am Chem Soc. Author manuscript; available in PMC 2009 October 27. 


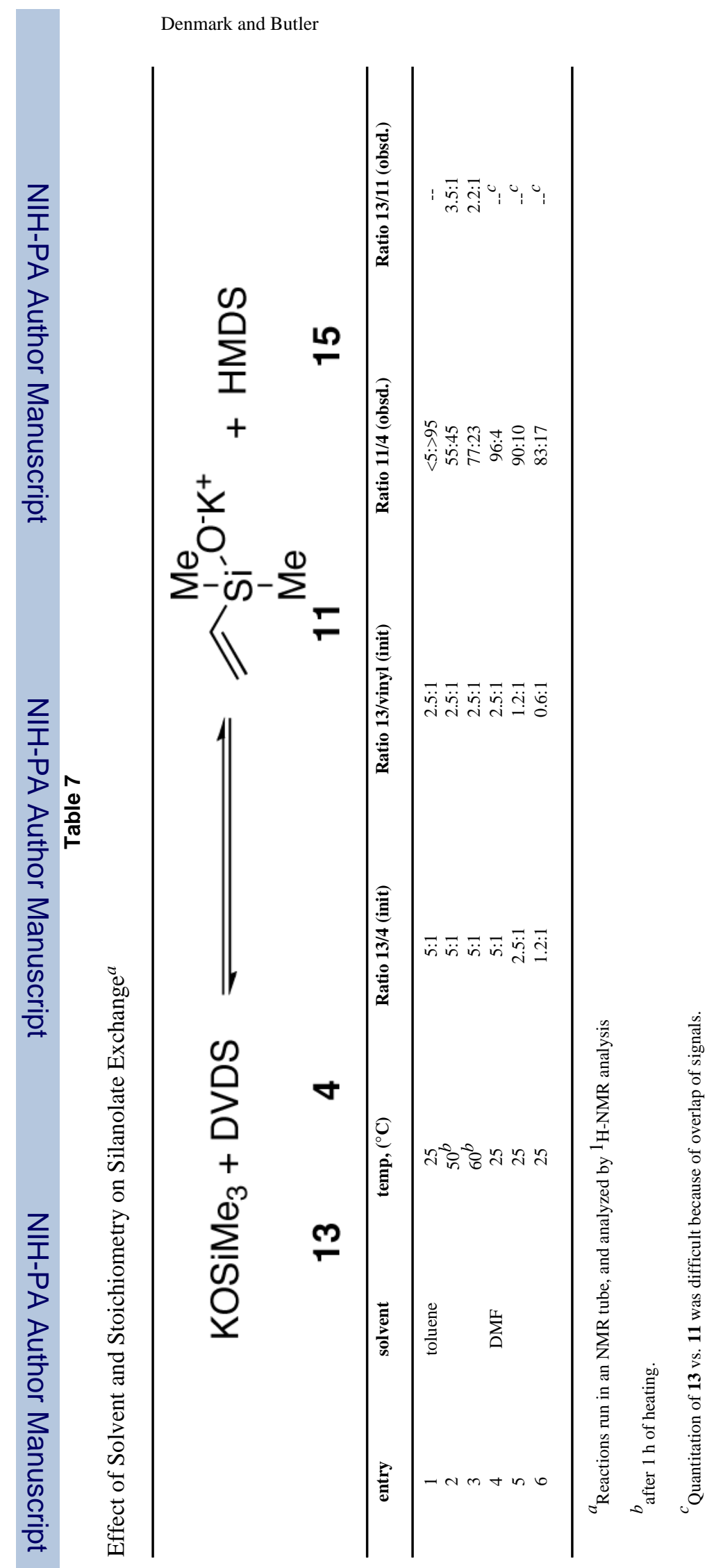

J Am Chem Soc. Author manuscript; available in PMC 2009 October 27. 
Table 8

Preparative Vinylation of Aryl Bromides using DVDS

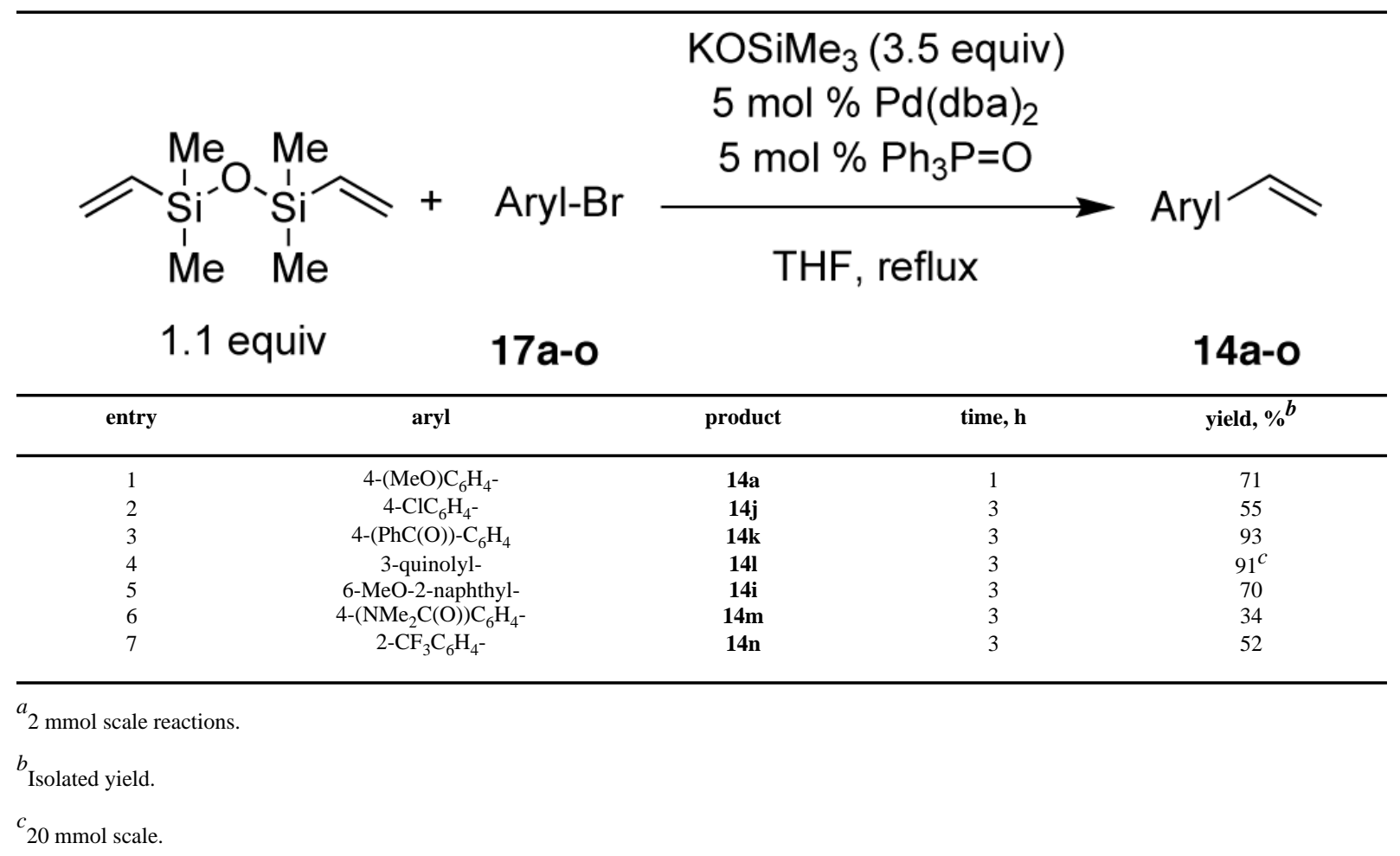




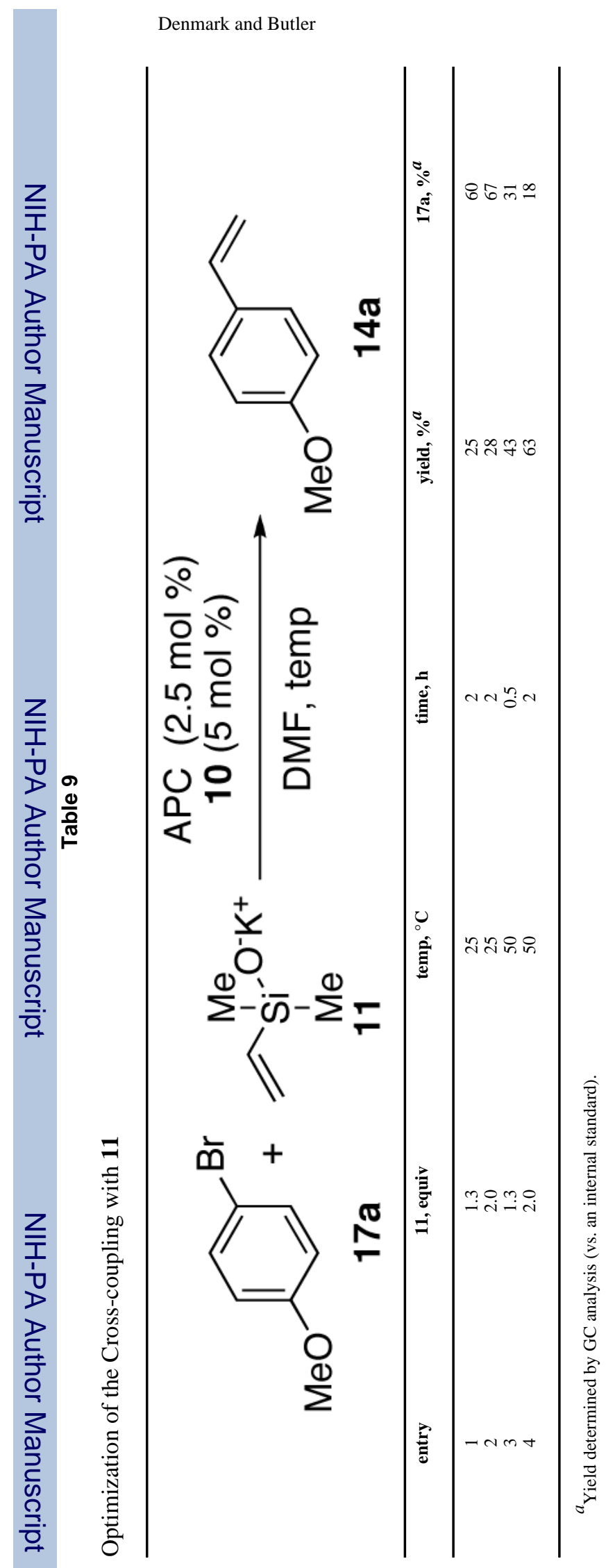

J Am Chem Soc. Author manuscript; available in PMC 2009 October 27. 


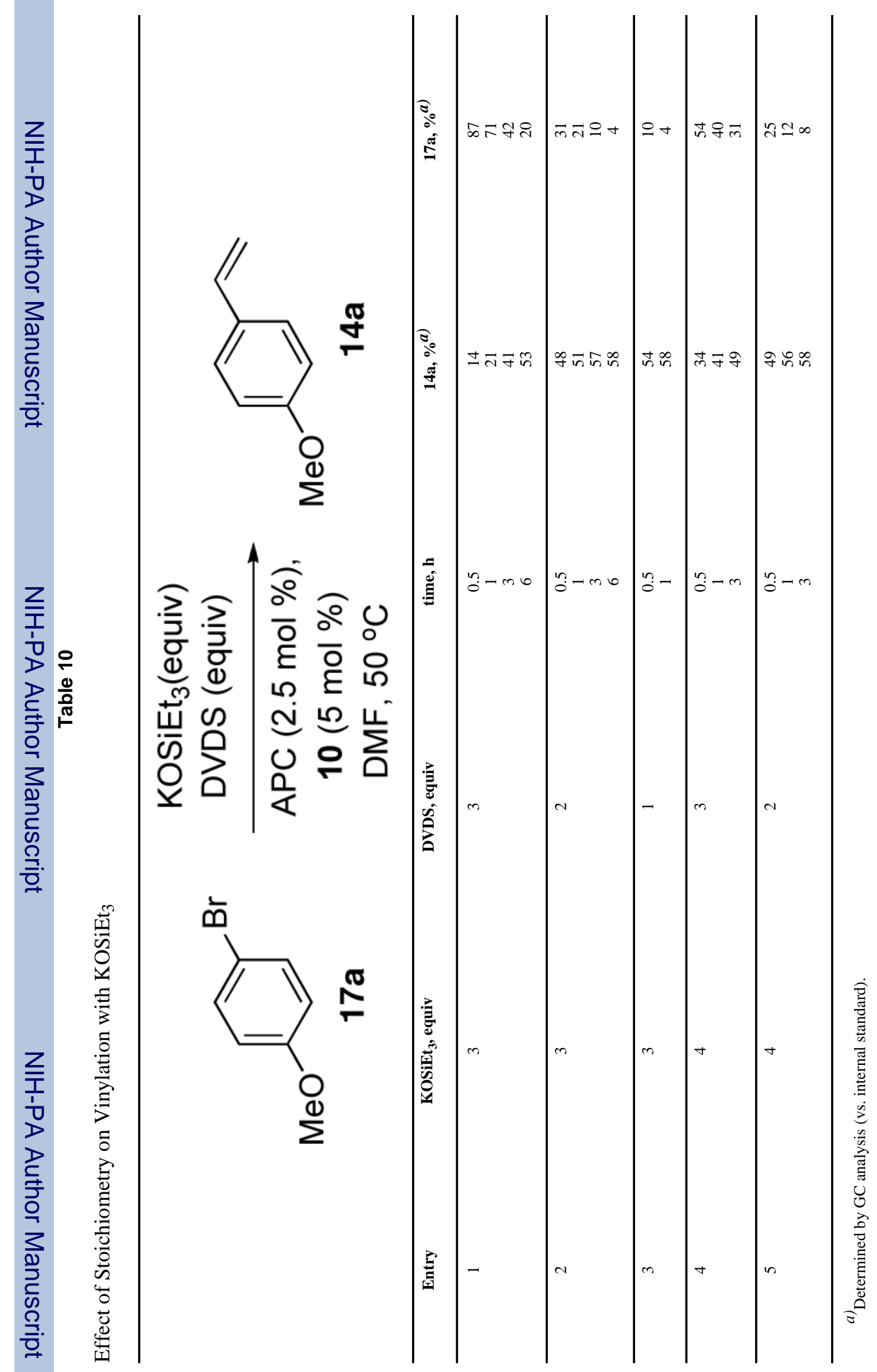

J Am Chem Soc. Author manuscript; available in PMC 2009 October 27. 


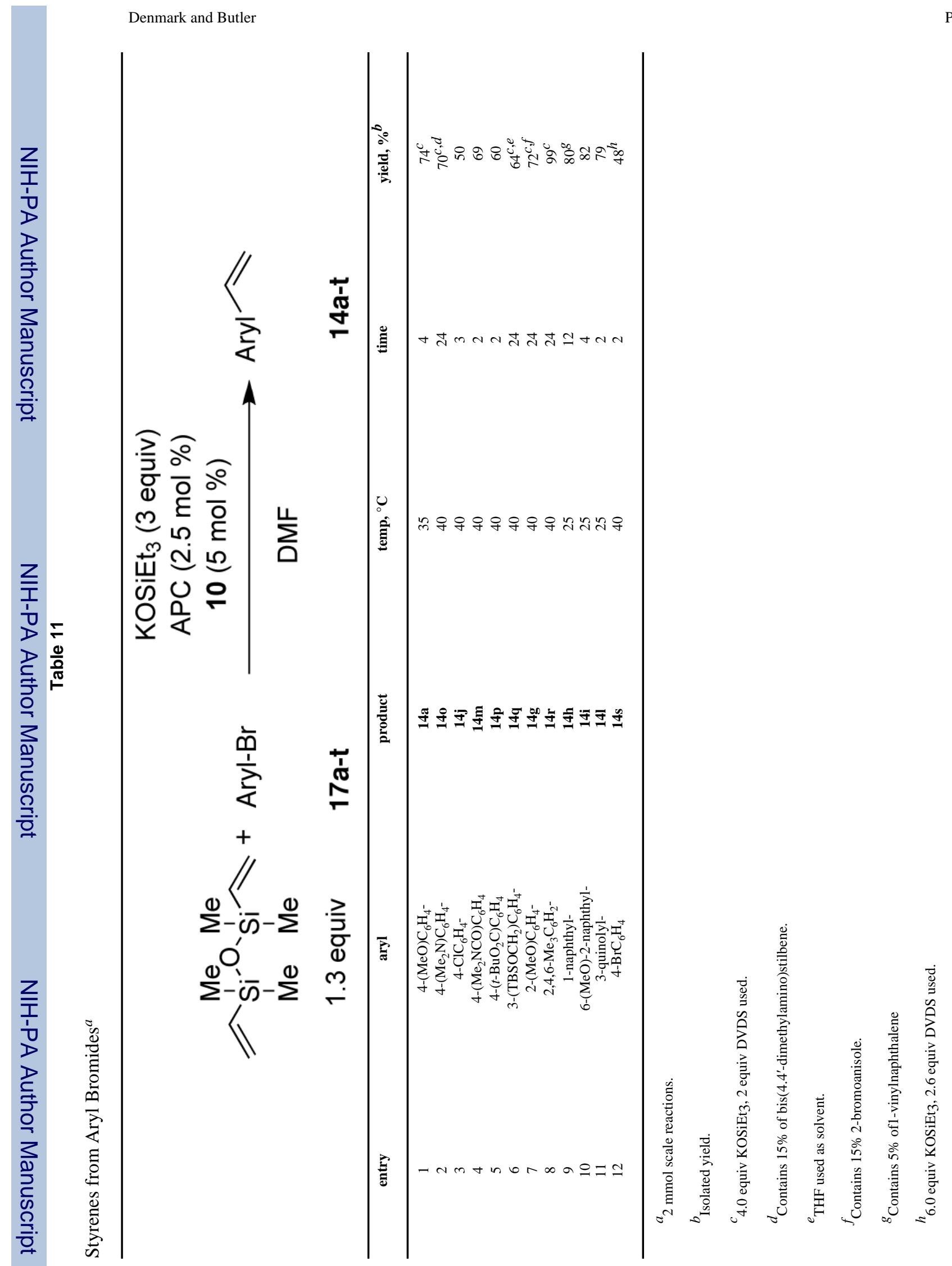

J Am Chem Soc. Author manuscript; available in PMC 2009 October 27. 